International Scientific Organization

http://iscientific.org/

Chemistry International

www.bosaljournals.com/chemint/

\title{
Aflatoxins biosynthesis, toxicity and intervention strategies: A review
}

\author{
Munawar Iqbal1, ${ }^{*}$, Mazhar Abbas ${ }^{2}$, Muhammad Adil² and Arif Nazir ${ }^{1}$
}

${ }^{1}$ Department of Chemistry, The University Lahore, Lahore Pakistan

${ }^{2}$ College of Veterinary \& Animal Sciences, Jhang (Sub-campus University of Veterinary and Animal Sciences, Lahore), Pakistan

*Corresponding author's E. mail: bosalvee@yahoo.com

\section{A R T I C L E I N F O}

\section{Article type:}

Review article

Article history:

Received December 2017

Accepted November 2018

July 2019 Issue

Keywords:

Aflatoxin

Biosynthesis

Toxicity

Control measures

\section{A B S T R A C T}

Aflatoxins (AFTs) are toxic products of fungal metabolism, associated with serious health consequences and substantial economic losses to agriculture, livestock and poultry sectors, particularly in the developing countries. This review outlines the current information on AFTs in terms of historical background, classification, relative occurrence and co-existence with other mycotoxins in various food commodities. The phenomenon of aflatoxin (AFT) biosynthesis has been elucidated with reference to molecular basis, genetic regulation and factors affecting the AFT production. Moreover, the in vivo disposition kinetics, toxicological action and toxico-pathological consequences of AFTs have also been highlighted. Currently employed strategies for the detection and detoxification of AFTs, biomarkers of exposure assessment, potential economic impact and regulatory considerations regarding the AFTs have been emphasized.

(c) 2019 International Scientific Organization: All rights reserved.

Capsule Summary: Aflatoxins biosynthesis, toxicity and intervention strategies along with in vivo disposition kinetics, toxicological action and toxico-pathological consequences are discussed in this article.

Cite This Article As: M. Iqbal, M. Abbas, M. Adil and A. Nazir. Aflatoxins biosynthesis, toxicity and intervention strategies: A review. Chemistry International 5(3) (2019) 168-191. https://doi.org/10.5281/zenodo.1570747

\section{INTRODUCTION}

\section{Molecular basis of AFT biosynthesis pathway}

The conversion of acetyl-CoA and malonyl-CoA catalyzed by fatty acid synthase-1 (Fas-1) and fatty acid synthase-2 (Fas2) provides the starter unit for AFT biosynthesis known as hexanoate (Minto and Townsend, 1997). Hexanoate is further acted upon by the iterative type I polyketide synthase to generate an intermediate compound called norsolorinic acid anthrone which on oxidation by the HypC enzyme forms the first stable intermediate, anthraquinone norsolorinic acid (Ehrlich et al., 2010). The sequential action of multiple enzymes including oxidoreductase, monooxygenase, dehydrogenase, flavin adenine dinucleotide-containing monooxygenase, esterase and versicolorin-B synthase leads to the production of versicolorin $B$. Versicolorin B desaturase catalyzes the conversion of versicolorin B into versicolorin A. The 2, 3 double bond present in the dihydrobisfuran ring of versicolorin A can be oxidized by the host enzyme to yield a reactive epoxide with carcinogenic, mutagenic and cytotoxic potential. Versicolorin A is converted to demethylsterigmatocystin followed by sterigmatocystin and then 0-methylsterigmatocystin through multiple enzymatic reactions and finally, $\mathrm{AFB}_{1}$ from 0 -methylsterigmatocystin is produced cytochrome P-450 monooxygenase OrdA action.

\section{Genetic regulation of AFT biosynthesis}


Table 1: Reported $\mathrm{LD}_{50} / \mathrm{LC}_{50}$ values of AFTs for different species of animals, birds and aquaculture

\begin{tabular}{lll}
\hline Animal & $\mathrm{LD}_{50}(\mathrm{mg} / \mathrm{kg})$ & References \\
\hline Rabbit & 0.3 & Newberne and Butler (1969) \\
Duckling & $0.3-0.6$ & Howard (1983) \\
Rainbow trout & 0.5 & Lovell (1989) \\
Cat & 0.55 & Newberne and Butler (1969) \\
Pig & 0.62 & Newberne and Butler (1969) \\
Mosquitofish & 0.68 & McKean et al. (2006b) \\
Cattle calf & $0.5-1.0$ & Wogen (1969) \\
Turkey & $0.5-1.0$ & Wogen (1969) \\
Dog & 1.0 & Newberne and Butler (1969) \\
Copepod & $1.0(\mathrm{LC} 50)$ & Reiss (1972b) \\
Guinea pig & $1.4-2.0$ & Howard (1983) \\
Horse & 2.0 & Wogen (1969) \\
Sheep & 2.0 & Armbrecht et al. (1970) \\
Monkey & 2.2 & Howard (1983) \\
Rat & $5.5-17.9$ & Howard (1983) \\
Chicken & 6.3 & Howard (1983) \\
Mouse & 9.0 & Newberne and Butler (1969) \\
Salmon & 10 & Schoental (1967) \\
Hamster & 10.2 & Robins and Richard (1992) \\
Catfish & $10-15$ & Jantrarotai et al. (1990) \\
Rohu & $12-13.3$ & Sahoo et al. (2003) \\
Brine shrimp & $14.0(\mathrm{LC}$ 50) & Reiss (1972a) \\
Nile tilapia & 100 & Tuan et al. (2002) \\
Pacific blue shrimp & 100.5 & Wiseman et al. (1982) \\
Pacific white shrimp & $50-300$ & Wiseman et al. (1982) \\
\hline
\end{tabular}

The biosynthesis of AFTs encompasses at least 25 genes for encoding the enzymes to catalyze 18 enzymatic reactions and associated regulatory pathways (Yu et al., 2004). The AFT pathway genes regulated by the regulatory gene, aflR, consist of $70 \mathrm{~kb}$ of fungal genome located in the cluster of genome (Yabe and Nakajima, 2004; Price et al., 2006). Flaherty and Payne, 1997 recorded up-regulated transcription of AFT pathway genes due to overexpression of aflR in A. flavus. The expression of other AFT pathway genes abolished following the deletion of aflR in A. parasiticus (Cary et al., 2000). AFT synthesis also requires the aflS (afl) gene and knockout of aflS deprived the mutant fungi of AFT synthesizing capacity (Meyers et al., 1998). Other genes like laeA and $v e A$ have been reported to exert a global regulatory function on $\mathrm{AF}$ biosynthesis (Bok and Keller, 2004; Calvo et al., 2004). The laeA gene encodes a nuclear protein called LaeA which triggers the transcription of gene clusters associated with AFT synthesis and secondary metabolism (Yu, 2012). Deletion of veA gene in A. flavus (A. flavus) and A. parasiticus (A. parasiticus) abolished the AFT formation (Yu, 2012).

\section{Factors affecting the AFT biosynthesis}

The AFT biosynthesis is affected by various biotic and abiotic factors. For the sake of convenience, these elements are categorized into biological, physiological, nutritional, environmental and agricultural factors.

\section{A) Biological factors}

Several biological factors including cultivar, soil type, viable fungal species in the soil and plant metabolites have been documented to influence the AFT formation.

\section{B) Cultivars}

Given that, the local varieties of maize and peanuts were relatively more susceptible to AFT contamination (Hell et al., 2003; Mutegi et al., 2009), the development of transgenic, AFT-resistant cultivars represented a promising strategy to reduce AFT contamination of crops. Contrary to typical peanuts, the transgenic variety exhibiting $\mathrm{Bt}$ (Bacillus thuringiensis) gene displayed considerably lower level of AFTs (Ozias-Akins et al., 2002). Thakare et al. (2017) employed the host-induced gene silencing and transformed 
maize by targeting the aflC gene, encoding an enzyme involved in AFT biosynthetic pathway.

\section{C) Soil type}

The survival of AFTs is greatly affected by the type of soil. Sandy loam soil led to rapid decomposition and shorter persistence of AFTs than silt loam and silty clay loam soils (Angle, 1986). Heavier soil with a high water-holding potential declined the level of AFT contamination. While light and sandy soil promoted the growth of A. flavus and thereby increased the likelihood of AF contamination (Torres et al., 2014).

\section{D) Viable fungal species inhabiting the soil}

Strain-specific variations in terms of AFT production have been documented for soil isolates of A. flavus. Horn and Dorner, (1999) reported relatively high levels of AFTs produced by the isolates of S-strain than the L-strain of $A$. flavus. The occurrence of S-strain of A. flavus was associated with the level of AFTs in peanuts while such correlation was not recorded for L-strain (Mutegi et al., 2012).

\section{E) Plant metabolites}

Plants synthesize the antimicrobial substances known as phytoalexins that affect the process of AFT synthesis (Greene-McDowelle et al., 1999). The resistance of immature peanut pods against fungal infection was attributed to phytoalexins (Vidhyasekaran et al., 1972) and peanuts challenged with certain species of fungi, including A. flavus synthesized the phytoalexins (Wotton and Strange, 1985; Dorner et al., 1989). Under specific conditions, $n$-decyl aldehyde diminished the growth of $A$. parasiticus and subsequent AFT formation by more than 95\% (Wright et al., 2000). Yu, (2012) documented that octanal and hexanal reduced the fungal growth by $60 \%$ and $50 \%$ respectively. However, octanal augmented the AFT formation by $500 \%$, while hexanal had no effect on AFT synthesis.

\section{Physiological factors}

AFT biosynthesis is also affected by certain physiological attributes including the culture $\mathrm{pH}$, developmental stage of crop and oxidative stress.

\section{A) Culture pH}

AFT production differs with variation in acid-base equilibrium ratio of the culture medium. Fungal AFT production increased by almost 5-10 times at the pH levels of 4 or 5 than pH 8 (Keller et al., 1997). Low pH led to activation of AFT-producing genes therefore acidic medium favored the AFT biosynthesis by A. flavus (Cotty, 1988; MarroquínCardona et al., 2014).

\section{B) Developmental stage of crop}

Lack of fungal sporulation and serial subculturing resulted in the loss of AFT-producing capacity (Bennett and Papa, 1988) and AFT synthesis was blocked by some compounds capable to inhibit the sporulation in Aspergillus nidulans (A. nidulans) and A. parasiticus (Reib, 1982). Likewise, smaller and immature kernels being deficient in phytoalexins were highly susceptible to A. flavus-induced invasion and AFT formation (Hill et al., 1983; Sanders et al., 1985).

\section{F) Oxidative stress}

Oxidative stress has been proposed to trigger AFT synthesis as an essential element of the fungal cellular response through the production of reactive oxygen species (Narasaiah et al., 2006; Reverberi et al., 2006; Roze et al., 2011). Jayashree and Subramanyam, (2000) regarded the oxidative stress as a prerequisite for AFT synthesis. Consequently, antioxidants including ascorbic acid, caffeic acid and hydrolysable tannins efficiently inhibited the pathway of AFT biosynthesis (Mahoney and Molyneux, 2004; Kim et al., 2008; Yu, 2012).

\section{Nutritional factors}

Nutritional sources including amino acids, carbon, nitrogen, lipids and trace elements have been documented to affect the AFT biosynthesis (Luchese and Harrigan, 1993; Cuero et al., 2003).

\section{A) Amino acids}

Current studies have demonstrated the variable effects of some amino acids on AFT biosynthesis. Media containing alanine, aspartate, asparagine, proline and glutamine favored the process of AF synthesis (Reddy et al., 1979). Payne et al., (1983) examined the effects of asparagine, ammonium sulphate, casein and proline on AFT production by $A$. flavus and A. Parasiticus. Proline triggered the AFT production per gram of mycelium than the other amino acids being investigated. Wilkinson et al., (2007) reported that tyrosine improved while tryptophan inhibited the AFT formation by $A$. flavus.

\section{B) Carbon}

Although lactose, sorbose and peptone did not influence the AFT synthesis, simple sugars such as glucose, fructose, maltose and sucrose promoted the AFT formation (Buchanan and Lewis, 1984). Woloshuk et al., (1997) documented the correlation between AFT synthesis and $\alpha$-amylase activity in A. flavus. Nevertheless, the molecular mechanism underlying the regulation of AFT gene pathway expression by carbon sources needs further research $(\mathrm{Yu}, 2012)$.

\section{C) Nitrogen}


Table 2: Reported $\mathrm{LD}_{50} / \mathrm{LC}_{50}$ values of AFTs for different species of animals, birds and aquaculture

\begin{tabular}{|c|c|c|c|}
\hline $\begin{array}{l}\text { Exposed } \\
\text { species }\end{array}$ & Dose of AFTs & Clinico-pathological effects & References \\
\hline $\begin{array}{l}\text { Hereford } \\
\text { cattle calves }\end{array}$ & $2230 \mathrm{ppb}$ & $\begin{array}{l}\text { Anorexia, depression, photosensitization, diarrhoea, jaundice, } \\
\text { elevated serum levels of bilirubin and hepatic enzymes, } \\
\text { hepatocyte damage and death }\end{array}$ & $\begin{array}{l}\text { McKenzie et al. } \\
\text { (1981) }\end{array}$ \\
\hline $\begin{array}{l}\text { Male Holstein } \\
\text { cattle calves }\end{array}$ & $1130 \mathrm{ppb}$ & $\begin{array}{l}\text { General unthriftiness, diarrhea, hydrothorax, lymphopenia, } \\
\text { monocytosis, megalocytosis, hepatic congestion with necrosis } \\
\text { and } 17 \% \text { mortality }\end{array}$ & $\begin{array}{l}\text { Kaleibar and } \\
\text { Helan (2013) }\end{array}$ \\
\hline Adult Cattle & $33500 \mathrm{ppb}$ & $\begin{array}{l}\text { Anorexia, depression, diarrhea, photosensitization, visceral } \\
\text { hemorrhages, blood exudation from natural orifices, prolapse, } \\
\text { anasarca and death }\end{array}$ & Umar et al. (2015) \\
\hline $\begin{array}{l}\text { Nili-Ravi } \\
\text { buffaloes }\end{array}$ & $\begin{array}{l}500 \mathrm{ppb} \text { per } \\
\text { animal per day }\end{array}$ & $\begin{array}{l}\text { Reduction of average daily feed intake and hematological } \\
\text { parameters, while elevation of serum biochemical parameters }\end{array}$ & $\begin{array}{l}\text { Akhtar et al. } \\
(2014)\end{array}$ \\
\hline \multirow[t]{2}{*}{$\begin{array}{l}\text { Nili-Ravi } \\
\text { buffalo } \\
\text { heifers }\end{array}$} & $\begin{array}{l}554 \text { ppm, } 953 \\
\text { ppm, } 2022 \mathrm{ppm}, \\
3202 \mathrm{ppm}\end{array}$ & $\begin{array}{l}\text { Concentrations of serum glucose, total protein and cholesterol } \\
\text { remain unaltered while concentration of serum urea was } \\
\text { significantly elevated }\end{array}$ & Aslam et al. (2014) \\
\hline & $2.5-6.2 \mathrm{ppm}$ & $\begin{array}{l}\text { Anorexia, dullness, lethargy, submandibular oedema, } \\
\text { enlargement and congestion of liver with centrolobular } \\
\text { necrosis, cellular vacuolation, cirrhosis and bile duct } \\
\text { hyperplasia }\end{array}$ & $\begin{array}{l}\text { Osman et al. } \\
(2004)\end{array}$ \\
\hline Camels & $\begin{array}{l}0.1-1.0 \mathrm{ppb} \text { and } \\
0.05-0.1 \mathrm{ppb}\end{array}$ & $\begin{array}{l}\text { Fatty degeneration, congestion and fibrosis of liver with } \\
\text { petechial hemorrhages, vacuolar degenerations, cholangitis, } \\
\text { cirrhosis, bile duct carcinoma and hepatocellular carcinoma } \\
\text { Anorexia, icterus, rapid weight loss immediately before death, } \\
\text { black colored liver with marked centrilobular hepatic necrosis }\end{array}$ & $\begin{array}{l}\text { Al-Hizab et al. } \\
(2015)\end{array}$ \\
\hline \multirow{2}{*}{$\begin{array}{l}\text { Mature } \\
\text { Arabian } \\
\text { horses }\end{array}$} & $58.4 \mathrm{ppb}$ & and bile duct hyperplasia, congestion of adrenal cortex & $\begin{array}{l}\text { Greene and } \\
\text { Oehme (1976) }\end{array}$ \\
\hline & $\begin{array}{l}0.075 \text { ppm (over } \\
36 \text { or } 39 \text { days), }\end{array}$ & $\begin{array}{l}\text { Anorexia, depression, generalized icterus, Prothrombin time, } \\
\text { total plasma bilirubin and the icteric index increased markedly }\end{array}$ & \\
\hline Adult male & $0.15 \mathrm{ppm}$ (over & before death, hemorrhages, brown to tan livers and dark & Cysewski et al. \\
\hline $\begin{array}{l}\text { Shetland } \\
\text { ponies }\end{array}$ & $\begin{array}{l}25 \text { or } 32 \text { days) } \\
\text { and } 0.3 \text { ppm } \\
\text { (over } 12 \text { or } 16 \\
\text { days) }\end{array}$ & $\begin{array}{l}\text { colored kidneys, centrolobular fatty change with hepatic-cell } \\
\text { necrosis and periportal fibrosis }\end{array}$ & $(1982)$ \\
\hline \multirow[t]{2}{*}{ Sheep } & $4 \mathrm{ppm}$ & $\begin{array}{l}\text { Anorexia, diarrhea, excessive salivation, ruminal atony, scour, } \\
\text { rectal prolapse, fever and death }\end{array}$ & $\begin{array}{l}\text { Wylie and } \\
\text { Morehouse (1978) }\end{array}$ \\
\hline & $0.75 \mathrm{ppm}$ & Anorexia, apathy, hepatic lesions, neurological signs and death & $\begin{array}{l}\text { Suliman et al. } \\
\text { (1987) }\end{array}$ \\
\hline $\begin{array}{l}\text { Crossbreed } \\
\text { goats }\end{array}$ & $\begin{array}{l}0.1 \mathrm{ppm} \text { for } 34 \\
\text { days; } 0.2 \text { ppm for } \\
18 \text { days; and } 0.4 \\
\text { ppm for } 10 \text { days }\end{array}$ & $\begin{array}{l}\text { Anorexia, loss of body weight, mucopurulent nasal discharge, } \\
\text { dyspnea, coughing, lethargy, icterus, diarrhea, elevated } \\
\text { hematological and serum biochemical parameters and } \\
\text { subnormal body temperature } 24 \text { to } 48 \text { hours before death }\end{array}$ & Clark et al. (1984) \\
\hline
\end{tabular}

Male goats $\quad 0.1 \mathrm{ppm}$ for 34 days; $0.2 \mathrm{ppm}$ for 18 days; and 0.4 ppm for 10 days White tailed $800 \mathrm{ppb}$ deer fawns for 8 weeks $667.0,11.65$, Bullfrogs
141.74 , and 3.53 ppm for 120 days
Pneumonia, rhinitis, nasal discharge, ascites, paleness of liver, petechial hemorrhages, icterus, bile duct proliferation, hepatocytic karyomegaly and hepatocellular degeneration

Diminished feed intake, body weight gain and liver functions

Absence of liver tumors, increased hepatocyte and biliary duct cell proliferation and appearance of basophilic hepatocytes
Miller et al. (1984)

Quist et al. (1997)

Grassi et al. (2007) 
Table 2: Continue...

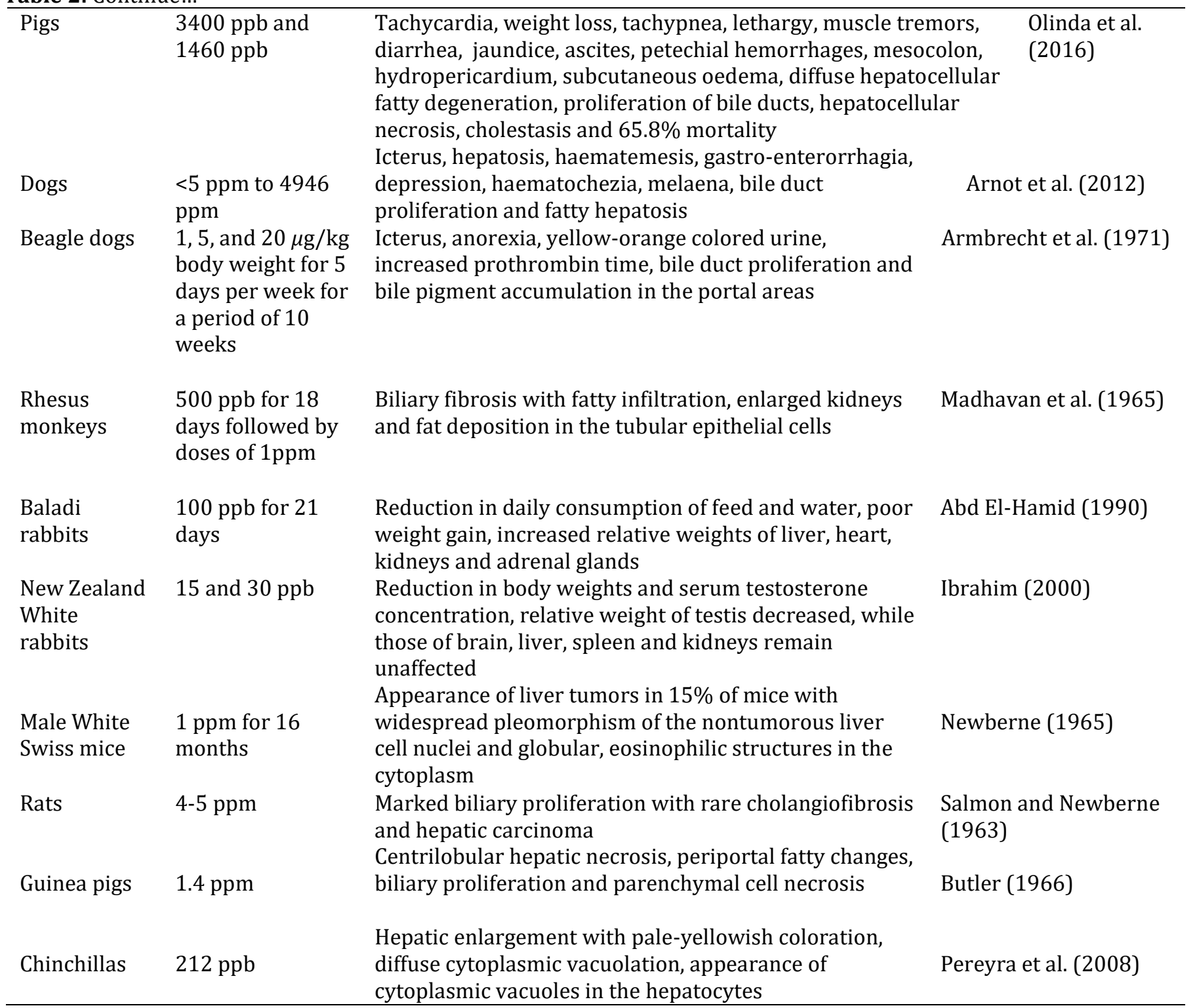

The causal relationship between nitrogen and AFT synthesis has been validated by nitrate-induced suppression of AFT production (Kachholz and Demain, 1983; Payne and Brown, 1998). Moreover, the addition of ammonium nitrite, ammonium nitrate and ammonium sulfate supported AFT production; whereas sodium nitrite and sodium nitrate had no impact on AFT synthesis (Reddy et al., 1979).

\section{D) Lipids}

Lipid substrate constitutes an excellent carbon source for AFT synthesis (Fanelli et al., 1995) and 0.5\% soybean oil induced the lipase gene expression followed by AFT formation (Yu et al., 2003). Saturated fatty acids (stearic acid, palmitic acid and myristic acid) stimulated while unsaturated fatty acids (linoleic acid and oleic acid) inhibited the AFT synthesis (Priyadarshini and Tulpule, 1980). However, Chulze et al., (1991) reported the stimulation of AFT production attributed to unsaturated fatty acids.

\section{Environmental factors}

The impact of environmental attributes like topography, climate and weather on AFT production has been markedly established.

\section{A) Topography}

A. flavus has been isolated from the soil of all climatic zones, but it is quite common in warm regions (latitudes 26-35) and relatively rare in areas with latitudes above $45^{\circ}$ (Klich, 2002). Aspergillus nomius (A. nomius) and $A$. parasiticus 
responsible to produce both B and G AFTs, are seldom found in some areas (Cotty and Cardwell, 1999). Consequently, the areas with latitudes below $35^{\circ}$ are more likely to come across the AFT contamination (Logrieco and Visconti, 2004).

\section{B) Climate}

Climatic conditions represent the main determinant of fungal invasion followed by AFT production (Magan et al., 2003) and acute aflatoxicosis with mortality has been attributed to climate change (Lewis et al., 2005). AFT producing fungi usually inhabit the tropical, warm arid and semi-arid regions and irrigated hot deserts with characteristic changes in climate (Bock et al., 2004). Hot and dry climate enhanced the susceptibility of maize to AFT contamination by facilitating the development, conidiation and dispersion of A. flavus and thereby attenuating the growth of affected maize (Scheidegger and Payne, 2003; Cotty and Jaime-Garcia, 2007; Chauhan et al., 2008; Magan et al., 2011).

\section{C) Weather}

Weather conditions reflected as short-term changes in temperature, rainfall pattern and relative humidity are recognized to affect the fungal AFT production (Miraglia et al., 2009; Marroquín-Cardona et al., 2014).

\section{D) Temperature}

Temperature is a major determinant that alters fungal growth and subsequent mycotoxin production (MarroquínCardona et al., 2014). Both low and high temperatures diminished the fungal viability and resultant AFT production (Miraglia et al., 2009; Paterson and Lima, 2011). Chilies produced during summer season in Pakistan, exhibited considerably high AFT content than those produced in winter (Iqbal et al., 2011). Generally, the optimal temperature for AFT synthesis ranged from $24^{\circ} \mathrm{C}$ to $30^{\circ} \mathrm{C}$ (Klich, 2007). However, OBrian et al., (2007) suggested a favorable temperature range of $28^{\circ} \mathrm{C}$ to $35^{\circ} \mathrm{C}$ while AFT production ceased as soon as temperature exceeded $36^{\circ} \mathrm{C}(\mathrm{Yu}, 2012)$.

\section{E) Drought}

Drought represents another modulating factor of AFT contamination. The xerophyte fungi, $A$. flavus and $A$. parasiticus are capable to grow and proliferate under drought condition. Drought stress deteriorated the natural immunity of crops against fungi through the reduction of phytoalexins synthesis (Dorner et al., 1989) and facilitated the AFT production by raising the proline content of exposed crops (Payne and Hagler, 1983).

\section{F) Rainfall}

Although adequate rainfall has been documented to impede or lessen the AFT contamination of peanuts, rain-fed conditions led to extensive AFT formation (Reddy et al., 2003). Besides, sorghum grown during monsoon season in India manifested comparatively high level of AFTs (Ratnavathi et al., 2012). Exposure to heavy rainfall has been implicated in AFT contamination of cottonseed and maize crops (Jaime-Garcia and Cotty, 2003; Lewis et al., 2005).

\section{G) Water activity}

Water activity (aw) also affects the fungal growth and AFT production (Marroquín-Cardona et al., 2014). Mousa et al., (2013) found the highest AFT production by A. flavus in polished rice at $21^{\circ} \mathrm{C}$ with aw range of 0.9-0.92 following an incubation period of 21 days. Peanuts synthesized adequate amount of phytoalexins at high aw $(>0.97)$, which was ultimately ceased at aw $<0.95$ (Dorner et al., 1989).

\section{H) Interactive effect of multiple weather elements on AFT synthesis}

In addition to the impact of individual weather elements, studies have also revealed the combined effect of various weather determinants on AFT biosynthesis. Drought and high temperature, promoted the AFT production (Bankole et al., 2006) and predisposed the peanuts (Craufud et al., 2006), transgenic Bt cottonseed (Bock and Cotty, 1999; Magan et al., 2011) and European maize (Paterson and Lima, 2011) to aflatoxicosis. Likewise, low rainfall with high ambient temperature enhanced the susceptibility of peanuts to aflatoxicosis (Chauhan et al., 2010; Paterson and Lima, 2010). Variations in environmental temperature and its interactive effect with water activity affected the expression pattern of AFT pathway regulatory genes (aflR and aflS) in A. parasiticus and A. flavus (Magan and Aldred, 2007; OBrian et al., 2007; Schmidt-Heydt et al., 2010). Gallo et al., (2016) recorded maximum fungal growth, $\mathrm{AFB}_{1}$ synthesis and upregulated expression levels of aflR and aflS genes at 0.96 aw and $28^{\circ} \mathrm{C}$, while $20^{\circ} \mathrm{C}$ and $37^{\circ} \mathrm{C}$ led to downregulation of AFT gene pathway.

\section{Agricultural factors}

Inappropriate agricultural systems, such as sowing time, tillage, crop rotation, irrigation, and application of fertilizers also contributed to A. flavus infestation followed by AFT production (Torres et al., 2014). Crop rotation, particularly comprising of fungi-resistant crops reduced the likelihood of between-season fungal viability (Mutegi et al., 2012). For instance, the rate of fungal infection and AFT synthesis enhanced when peanuts were continuously grown on the same land (Ortiz et al., 2011). Kebede et al., (2012) demonstrated the effectiveness of irrigation for diminshing plant stress to counteract the AFT problem. Insect damage triggerd the fungal penetration and AFT contamination in affected cereals, nuts, crops and other food commodities. 
Table 3: Reported clinico-pathological effects associated with aflatoxicosis in different avian species

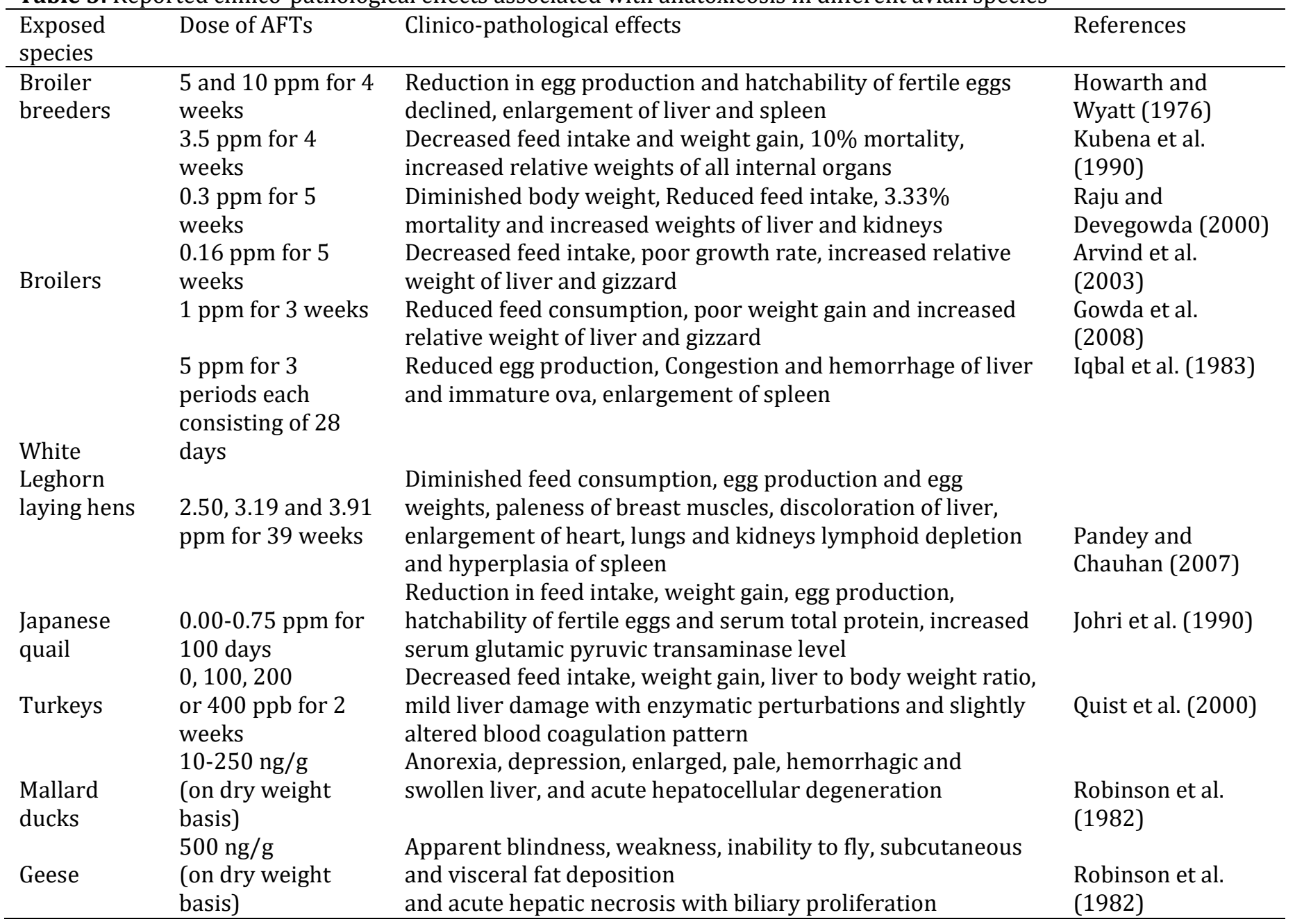

Waliyar et al., (2008) observed high AFT concentration in peanuts exposed to insect damage. Fungicides and insecticides have been successfully employed in the field to avert fungal growth and insect damage thereby preventing the AFT biosynthesis (D’Mello et al., 1998; Dorner et al., 2003). Application of insecticide during the cultivation of peanuts led to remarkable reduction in A. flavus infestation and AFT production (Bowen and Mack, 1993).

Even though, harvesting should be preferably done at a proper time, following the maturation of crops. However, earlier harvesting is recommended for some nuts to evade potential hull splitting and insect damage which reinvigorate the fungal infection. Timely harvesting of fruits with subsequent cooling is critical to minimize the risk of fungal invasion (Marroquín-Cardona et al., 2014). Inappropriate threshing and digging practices enhanced the vulnerability of peanuts to fungal infection and ensuing AFT contamination because of mechanical damage to kernels (Heathcote and Hibbert, 1978). Post-harvest AFT contamination of peanuts could be effectively controlled through proper drying with the maintenance of a safe humidity level (Torres et al., 2014) and segregation followed by the exclusion of contaminated peanuts (Dorner, 2008).

\section{Mechanism of action of AFTs}

The cytotoxic and carcinogenic potential of AFT metabolite, $\mathrm{AFB}_{1}$ has been clearly established. Studies have reported the induction of lipid peroxidation followed by oxidative injury to hepatic tissue of rats and inhibition of cyclic nucleotide phosphodiesterase activity in liver, heart, brain and kidney tissues by $\mathrm{AFB}_{1}$ (Shen et al., 1995; Bonsi et al., 1999). The reactive metabolite of $\mathrm{AFB}_{1}\left(\mathrm{AFB}_{1}-8\right.$, 9-epoxide) binds with $\mathrm{N}_{7}$ of guanine through covalent linkage (Lillehoj, 1991) to yield $\mathrm{AFB}_{1}-\mathrm{N}_{7}$-guanine adducts in exposed cells (Bailey, 1994). These $\mathrm{AFB}_{1}-\mathrm{N}_{7}$-guanine adducts have been implicated to instigate $\mathrm{G}$ to $\mathrm{T}$ transversions, mutations and tumor formation (Foster et al., 1983). The $G$ to $T$ transversion occurring at codon 249 of p53 tumor suppressor gene has been linked with human hepatocellular carcinomas (Wang and Groopman, 1999). 
Table 4: AFTs detection methods in food and feed items

\begin{tabular}{|c|c|c|c|c|c|c|}
\hline $\begin{array}{l}\text { AFB1, } \\
\text { AFB2, } \\
\text { AFG1, } \\
\text { AFG2 }\end{array}$ & $\begin{array}{l}\text { Cereals, } \\
\text { Baby } \\
\text { food }\end{array}$ & $\begin{array}{l}\text { Acetonitril:w } \\
\text { ater } \\
(80: 20)\end{array}$ & $\begin{array}{l}\text { BEH C18 Analytical } \\
(1.7 \mu \mathrm{m}, 2.1 \times 50 \mathrm{~mm}) \text {, } \\
\text { (Waters) } \\
\text { MS-TQD }^{\mathrm{TM}}, \mathrm{R}^{-}\end{array}$ & $\begin{array}{l}\mathrm{H}_{2} \mathrm{O}+\mathrm{NH}_{4} \mathrm{Ac}+\mathrm{HCO} \\
\mathrm{OH} \\
300 \mu \mathrm{L} / \mathrm{min}\end{array}$ & $\begin{array}{l}0.1 /-\quad \mu \mathrm{g} / \mathrm{Kg} \\
\text { (cereals) } \\
0.025 /-\mu \mathrm{g} / \mathrm{Kg} \\
\text { (infant) }\end{array}$ & $\begin{array}{l}\text { Beltran et al. } \\
(2011)\end{array}$ \\
\hline $\begin{array}{l}\text { AFB1, } \\
\text { AFB2, } \\
\text { AFG1, } \\
\text { AFG2 }\end{array}$ & $\begin{array}{l}\text { Dried } \\
\text { fruit } \\
\text { and } \\
\text { Beans }\end{array}$ & $\begin{array}{l}\text { Methanol: } \\
\text { water } \\
(80: 20)+1 \mathrm{~g}\end{array}$ & $\begin{array}{l}\text { Intercell ODS-3 }(5 \mu \mathrm{m}, \\
4.6 \times 250 \mathrm{~mm}) \text { GL Science, } \\
\text { Tokyo, Japan. } \\
\text { FD(365-450nm), Rt- }\end{array}$ & $\begin{array}{l}\text { acetonitril } \\
\text { :methanol:Water } \\
(8: 27: 65) \\
0.8 \mathrm{~mL} / \mathrm{min}\end{array}$ & $\begin{array}{l}0.5 /- \\
1.0 /-\quad \mu g / K g \\
0.5 /- \\
1.0 /-\end{array}$ & $\begin{array}{l}\text { Lutfullah } \\
\text { and Hussain } \\
\text { (2011) }\end{array}$ \\
\hline $\begin{array}{l}\text { AFB1, } \\
\text { AFB2, } \\
\text { AFG1, } \\
\text { AFG2 }\end{array}$ & $\begin{array}{l}\text { Herbs, } \\
\text { Plants, } \\
\text { Tea }\end{array}$ & $\begin{array}{l}\text { Methanol: } \\
\text { water }(8: 2)\end{array}$ & $\begin{array}{l}\text { RP C-18 }(5 \mu \mathrm{m}, \\
4.6 \times 250 \mathrm{~mm}) \\
\text { FD }(360-435 \mathrm{~nm}), \mathrm{Rt}^{-}\end{array}$ & $\begin{array}{l}\text { Water: } \\
\text { acetonitril:methan } \\
\text { ol } \\
(6: 2: 3), 1 \mathrm{~mL} / \mathrm{min}\end{array}$ & $\begin{array}{l}0.5 / 1.5 \\
0.2 / 0.6 \\
\mu \mathrm{g} / \mathrm{Kg} \\
0.5 / 1.5 \\
0.2 / 0.6\end{array}$ & $\begin{array}{l}\text { Romagnoli } \\
\text { and others } \\
(2007)\end{array}$ \\
\hline $\begin{array}{l}\text { AFB1, } \\
\text { AFB2, } \\
\text { AFG1, } \\
\text { AFG2 }\end{array}$ & $\begin{array}{l}\text { Maize, } \\
\text { Rice }\end{array}$ & $\begin{array}{l}\text { Acetonitril:w } \\
\text { ater } \\
(99: 1)\end{array}$ & $\begin{array}{l}\text { RP C- } 18(10 \mu \mathrm{m}, \\
4.6 \times 250 \mathrm{~mm}) \\
\text { FD(360-450nm), Rt- }\end{array}$ & $\begin{array}{l}\text { methanol:acetonit } \\
\text { ril :Water } \\
(20: 20: 60) \\
1 \mathrm{~mL} / \mathrm{min},\end{array}$ & $\begin{array}{l}0.05 /- \\
0.0074 /-\mu \mathrm{g} / \mathrm{Kg} \\
0.1 /- \\
0.0074 /-\end{array}$ & $\begin{array}{l}\text { Liu et al. } \\
\text { (2006) }\end{array}$ \\
\hline $\begin{array}{l}\text { AFB1, } \\
\text { AFB2, } \\
\text { AFG1, } \\
\text { AFG2 }\end{array}$ & $\begin{array}{l}\text { Dried } \\
\text { fruits, } \\
\text { Nuts }\end{array}$ & $\begin{array}{l}\text { Methanol: } \\
\text { water } \\
(80: 20)+1 \mathrm{~g}\end{array}$ & $\begin{array}{l}\text { Intercell ODS- } 3(5 \mu \mathrm{m}, \\
4.6 \times 250 \mathrm{~mm}) \mathrm{GL} \text { Science, } \\
\text { Tokyo, Japan. } \\
\text { FD }(365-450 \mathrm{~nm}), \mathrm{R}^{-}\end{array}$ & $\begin{array}{l}\text { acetonitril } \\
\text { :methanol:Water } \\
(8: 27: 65) \\
0.8 \mathrm{~mL} / \mathrm{min}\end{array}$ & $\begin{array}{l}0.5 /- \\
1.0 /- \\
0.5 /- \\
1.0 /-\end{array}$ & $\begin{array}{l}\text { Luttfullah } \\
\text { and Hussain } \\
\text { (2011) }\end{array}$ \\
\hline $\begin{array}{l}\text { AFB1, } \\
\text { AFB2, } \\
\text { AFG1, } \\
\text { AFG2 }\end{array}$ & $\begin{array}{l}\text { Sorghu } \\
\text { m, } \\
\text { Pistachi } \\
\text { o }\end{array}$ & $\begin{array}{l}\text { Methanol: } \\
\text { water } \\
(80: 20)\end{array}$ & $\begin{array}{l}\text { ODS2-Spherisorb }(5 \mu \mathrm{m}, \\
4.6 \times 250 \mathrm{~mm})(\text { capital HPLC } \\
\text { Ltd) } \\
\mathrm{FD}(365-435 \mathrm{~nm}), \\
\mathrm{R}_{\mathrm{t}}=(8.8,7.6,6.5,5.8) \mathrm{min}\end{array}$ & $\begin{array}{l}\text { methanol:acetonit } \\
\text { ril:Water } \\
(20: 20: 60) \\
1 \mathrm{~mL} / \mathrm{min}\end{array}$ & $\begin{array}{l}0.03 / 0.16 \\
0.03 / 0.08 \\
0.03 / 0.08 \\
\mu \mathrm{g} / \mathrm{Kg} \\
0.03 / 0.08\end{array}$ & $\begin{array}{l}\text { Ghali et al. } \\
\text { (2009) }\end{array}$ \\
\hline $\begin{array}{l}\text { AFB1, } \\
\text { AFB2, } \\
\text { AFG1, } \\
\text { AFG2 } \\
\text { AFM1 } \\
\text { AFM2 }\end{array}$ & $\begin{array}{l}\text { Egg, } \\
\text { Milk, } \\
\text { Meat }\end{array}$ & $\begin{array}{l}\text { Acetone:wat } \\
\text { er } \\
(100: 100) \text {, } \\
\text { Chloroform }\end{array}$ & $\begin{array}{l}\text { Thermo LC-Si } \\
(4.6 \times 250 \mathrm{~mm}) \\
\text { FD }(365-435 \mathrm{~nm})\end{array}$ & $\begin{array}{l}\text { Toluene:ethylacet } \\
\text { at:formic } \\
\text { acid:methanol } \\
(90: 5: 2.2: 2.5) \\
2 \mathrm{~mL} / \mathrm{min}\end{array}$ & $\begin{array}{l}0.05 /- \\
0.05 /- \\
0.05 /-\mu g / \mathrm{Kg} \\
0.05 /- \\
0.05 /- \\
0.05 /-\end{array}$ & $\begin{array}{l}\text { Herzallah } \\
(2009)\end{array}$ \\
\hline $\begin{array}{l}\text { AFB1, } \\
\text { AFB2 }\end{array}$ & $\begin{array}{l}\text { Pistachi } \\
\text { o }\end{array}$ & $\begin{array}{l}\text { Methanol: } \\
\text { water } \\
(80: 20)+5 \mathrm{~g} \\
\mathrm{NaCl}\end{array}$ & $\begin{array}{l}\text { ODS- }(4.6 \times 250 \mathrm{~mm}) \\
\text { FD }(362-450 \mathrm{~nm})\end{array}$ & $\begin{array}{l}\text { Water:methanol:a } \\
\text { cetonitril } \\
(60: 20: 20)+350 \mu \mathrm{L} \\
4 \mathrm{M} \mathrm{HNO} 3 \\
1 \mathrm{~mL} / \mathrm{min}\end{array}$ & & $\begin{array}{l}\text { Sheibani and } \\
\text { Ghaziaskar } \\
\text { (2009) }\end{array}$ \\
\hline $\begin{array}{l}\text { AFB1, } \\
\text { AFB2, } \\
\text { AFG1, } \\
\text { AFG2 }\end{array}$ & Peanuts & $\begin{array}{l}\text { Methanol: } \\
\text { water }(80: 20\end{array}$ & $\begin{array}{l}\text { HPLC Cat }(5 \mu \mathrm{m} \text {, } \\
4.6 \times 250 \mathrm{~mm}) \\
\operatorname{FD}(365-435 \mathrm{~nm}), \\
\operatorname{AR}_{\mathrm{t}}(9.09,10.41,11.46,13.38 \\
) \min \\
\mathrm{BR}_{\mathrm{t}}(12.03,14.05,15.82,18.5 \\
8) \mathrm{min} \\
\mathrm{CR}_{\mathrm{t}}(28.34,03,33.08,38.74,4 \\
6.39) \mathrm{min}\end{array}$ & $\begin{array}{l}\text { acetonitril:Water: } \\
\text { methanol } \\
\mathrm{A}(23: 54: 23), 0.4 \mathrm{~m} \\
\mathrm{~L} / \mathrm{min}>\mathrm{LOD} \\
\mathrm{B}(17: 54: 29), 1 \mathrm{~mL} / \\
\mathrm{min}<\mathrm{Rt} \\
\mathrm{C}(8: 54: 38), 1 \mathrm{~mL} / \\
\mathrm{min}=<<\mathrm{Rt}\end{array}$ & $\begin{array}{l}0.03 / 0.1 \\
0.01 / 0.04 \\
(\mathrm{ng} / \mathrm{L}) \\
0.09 / 0.3 \\
0.06 / 0.2\end{array}$ & $\begin{array}{l}\text { Afsah-Hejri } \\
\text { et al. (2011) }\end{array}$ \\
\hline $\begin{array}{l}\text { AFB1, } \\
\text { AFB2, } \\
\text { AFG1, } \\
\text { AFG2 }\end{array}$ & Peprika & $\begin{array}{l}\text { Methanol: } \\
\text { water } \\
(60: 40)\end{array}$ & $\begin{array}{l}\text { RP-LiChrosorb C18 }(5 \mu \mathrm{m} \text {, } \\
4.6 \times 250 \mathrm{~mm}), \text { Merck, } \\
\text { Germany } \\
\text { FD }(365-435 \mathrm{~nm}) \\
\text { Rt }\end{array}$ & $\begin{array}{l}\text { methanol:acetonit } \\
\text { ril:Water } \\
(20: 20: 60) \\
1 \mathrm{~mL} / \mathrm{min}\end{array}$ & $\begin{array}{l}0.09 / 0.23 \\
0.09 / 0.23 \\
\mu \mathrm{g} / \mathrm{Kg} \\
0.14 / 0.45 \\
0.14 / 0.45\end{array}$ & $\begin{array}{l}\text { Shundo et al. } \\
\text { (2009 }\end{array}$ \\
\hline
\end{tabular}


Table 4: Continue...

\begin{tabular}{|c|c|c|c|c|c|c|}
\hline AFTs & Food & Extraction & $\begin{array}{l}\text { Colum, detector, retention } \\
\text { time }\end{array}$ & Solvent, Flow rate & LOD/LOQ & References \\
\hline AFB1 & $\begin{array}{l}\text { Cereal } \\
\text { Breakfa } \\
\text { st }\end{array}$ & $\begin{array}{l}\text { Methanol: } \\
\text { water } \\
(80: 20)\end{array}$ & $\begin{array}{l}\text { C } 18 \text { Nova-Pak } 60 \mathrm{~A},(4 \mu \mathrm{m}, \\
46 \times 250 \mathrm{~mm}) \\
\mathrm{FD}(335,465 \mathrm{~nm}) \\
\mathrm{R}_{\mathrm{t}}=5.85 \mathrm{~min}\end{array}$ & $\begin{array}{l}\text { Water:acetonitril: } \\
\text { methanol (20:4:3), } \\
1 \mathrm{~mL} / \mathrm{min}\end{array}$ & $0.05 / 4.33 \mathrm{ngg}^{-1}$ & $\begin{array}{l}\text { Villa and } \\
\text { Markaki } \\
(2009)\end{array}$ \\
\hline $\begin{array}{l}\text { AFB1, } \\
\text { AFM1 }\end{array}$ & $\begin{array}{l}\text { Breast } \\
\text { milk }\end{array}$ & $\begin{array}{l}\text { Nacl+Chlorof } \\
\text { orm } \\
(0.4 \mathrm{~mL}+2.4 \mathrm{~m} \\
\text { L) }\end{array}$ & $\begin{array}{l}\text { ODS2-Spherisorb ( } 5 \mu \mathrm{m}, \\
4.6 \times 25 \mathrm{~mm})(\text { capital HPLC } \\
\mathrm{Ltd}), \mathrm{FD}(365-418 \mathrm{~nm}) \\
\mathrm{R}_{\mathrm{t}}=3.46 \mathrm{~min}, \mathrm{R}_{\mathrm{t}}=2.83 \mathrm{~min}\end{array}$ & $\begin{array}{l}\text { methanol:acetonitr } \\
\text { il :Water } \\
(25: 50: 25) \\
1 \mathrm{~mL} / \mathrm{min}\end{array}$ & $5 /-\quad(\mathrm{ng} / \mathrm{L})$ & $\begin{array}{l}\text { Gurbay et al. } \\
(2006)\end{array}$ \\
\hline AFB1 & Wheat & Methanol & $\begin{array}{l}\text { Capcell-Pak C18 UG } 80 \\
(5 \mu \mathrm{m}, 4.6 \times 250 \mathrm{~mm}) \\
(\text { Shiseido, Japan }) \\
\text { FD(474-484nm), } \\
\mathrm{R}_{\mathrm{t}}=8.9 \mathrm{~min}\end{array}$ & $\begin{array}{l}\text { acetonitril :Water } \\
(25: 75), 1 \mathrm{~mL} / \mathrm{min}\end{array}$ & $0.14 / .74(\mu \mathrm{g} / \mathrm{L})$ & $\begin{array}{l}\text { Giray et al. } \\
(2007)\end{array}$ \\
\hline $\begin{array}{l}\text { AFB1, } \\
\text { AFB2, } \\
\text { AFG1, } \\
\text { AFG2 }\end{array}$ & Cocoa & $\begin{array}{l}\text { Methanol:wat } \\
\text { er }(8: 2)\end{array}$ & $\begin{array}{l}\text { Shimpack }(4.6 \times 250 \mathrm{~mm}) \\
\text { FD }(362-455 \mathrm{~nm}), 425 \mathrm{~nm} \text {, }\end{array}$ & $\begin{array}{l}\text { Water:acetonitril: } \\
\text { methanol } \\
(6: 2: 3), 1 \mathrm{~mL} / \mathrm{min}\end{array}$ & $0.001 /-(\mu \mathrm{g} / \mathrm{L})$ & $\begin{array}{l}\text { Copetti et al. } \\
\text { (2011) }\end{array}$ \\
\hline $\begin{array}{l}\text { AFB1, } \\
\text { AFB2, } \\
\text { AFG1, } \\
\text { AFG2 }\end{array}$ & $\begin{array}{l}\text { Red } \\
\text { Pepper }\end{array}$ & $\begin{array}{l}\text { Methanol:wat } \\
\text { er }(8: 2)\end{array}$ & $\begin{array}{l}\text { Ascentic } C_{18} \\
(4.6 \times 250 \mathrm{~mm}),(\text { supelco }) \\
\mathrm{FD}(360-440,465 \mathrm{~nm}), \\
\mathrm{R}_{\mathrm{t}}=(12.4,10.5,9.2,7.9) \mathrm{min}\end{array}$ & $\begin{array}{l}\text { methanol:acetonitr } \\
\text { il :Water } \\
(5: 2: 3), 1 \mathrm{~mL} / \mathrm{min} \text {, }\end{array}$ & $\begin{array}{l}0.05 /-\quad(\mathrm{mg} / \mathrm{Kg}) \\
0.02 /- \\
0.06 /- \\
0.06 /-\end{array}$ & $\begin{array}{l}\text { Cheraghali et } \\
\text { al. (2007) }\end{array}$ \\
\hline $\begin{array}{l}\text { AFB1, } \\
\text { AFB2, } \\
\text { AFG1, } \\
\text { AFG2 }\end{array}$ & $\begin{array}{l}\text { Pistachi } \\
\text { o Nuts }\end{array}$ & $\begin{array}{l}\text { Methanol:wat } \\
\text { er }(3: 2)+5 \mathrm{~g} \\
\text { Nacl/250mL } \\
\text { Extract }\end{array}$ & $\begin{array}{l}\text { Ascentic } \mathrm{C}_{18} \\
(4.6 \times 250 \mathrm{~mm}),(\text { supelco) } \\
\mathrm{FD}(360-440,465 \mathrm{~nm}) \\
\mathrm{R}_{\mathrm{t}}=(12.4,10.5,9.2,7.9) \mathrm{min}\end{array}$ & $\begin{array}{l}\text { methanol:acetonitr } \\
\text { il :Water } \\
(5: 2: 3), 1 \mathrm{~mL} / \mathrm{min} \text {, }\end{array}$ & $\begin{array}{l}0.06 /-\quad(\mathrm{mg} / \mathrm{Kg}) \\
0.03 /- \\
0.08 /- \\
0.07 /-\end{array}$ & $\begin{array}{l}\text { Cheraghali et } \\
\text { al. (2007) }\end{array}$ \\
\hline $\begin{array}{l}\text { AFB1, } \\
\text { AFB2, } \\
\text { AFG1, } \\
\text { AFG2 }\end{array}$ & $\begin{array}{l}\text { Chilli } \\
\text { powder }\end{array}$ & $\begin{array}{l}\text { Methanol: } \\
\text { water } \\
(80: 20)\end{array}$ & $\begin{array}{l}\text { Hichrome Hipersil H5ODS } \\
\text { C18 }(4.6 \times 250 \mathrm{~mm}) \\
\text { FD }(365-450 \mathrm{~nm}), \mathrm{R}^{-}\end{array}$ & $\begin{array}{l}\text { acetonitril } \\
\text { :methanol :Water } \\
(250: 125: 600), 1.5 \\
\text { mL/min, }\end{array}$ & $\begin{array}{l}0.1 /-\quad(\mu \mathrm{g} / \mathrm{Kg}) \\
0.1 /- \\
0.1 /- \\
0.1 /-\end{array}$ & $\begin{array}{l}\text { O' Riordan } \\
\text { and } \\
\text { Wilkinson } \\
(2009)\end{array}$ \\
\hline $\begin{array}{l}\text { AFB1, } \\
\text { AFB2, } \\
\text { AFG1, } \\
\text { AFG2 }\end{array}$ & $\begin{array}{l}\text { Cassava } \\
\text {,Yam } \\
\text { Chips }\end{array}$ & $\begin{array}{l}\text { Methanol: } \\
\text { water } \\
(85: 15)\end{array}$ & $\begin{array}{l}\text { Supelcosil LC-C } 18 \\
(4.6 \times 150 \mathrm{~mm}, 3 \mu \mathrm{m}) \\
\text { Supelco, USA. } \\
\text { FD(335-440nm), Rt- }\end{array}$ & $\begin{array}{l}\text { methanol } \\
: \text { Water:acetic acid } \\
(45: 55: 2), 1 \mathrm{~mL} / \mathrm{mi} \\
\mathrm{n},\end{array}$ & $\begin{array}{l}0.1 /-\quad(\mu \mathrm{g} / \mathrm{Kg}) \\
0.1 /- \\
0.1 /- \\
0.1 /-\end{array}$ & $\begin{array}{l}\text { Basaran and } \\
\text { Akhan } \\
(2008)\end{array}$ \\
\hline $\begin{array}{l}\text { AFB1, } \\
\text { AFB2, } \\
\text { AFG1, } \\
\text { AFG2 }\end{array}$ & Spices & $\begin{array}{l}\text { Methanol: } \\
\text { water } \\
(70: 30)\end{array}$ & 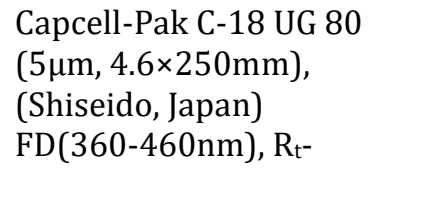 & $\begin{array}{l}\text { Water:acetonitril } \\
(90: 10), 1 \mathrm{~mL} / \mathrm{min}\end{array}$ & $\begin{array}{l}0.01 / 0.03 \\
0.01 / 0.0 .3 \\
(\mu \mathrm{g} / \mathrm{Kg}) \\
0.15 / 0.45 \\
0.02 / 0.06\end{array}$ & $\begin{array}{l}\text { Cho et al. } \\
(2008)\end{array}$ \\
\hline $\begin{array}{l}\text { AFB1, } \\
\text { AFB2, } \\
\text { AFG1, } \\
\text { AFG2 }\end{array}$ & $\begin{array}{l}\text { Figs, } \\
\text { Apricot }\end{array}$ & $\begin{array}{l}\text { Methanol:wat } \\
\text { er } \\
(100: 150)+4 \\
\text { g NaCl }\end{array}$ & $\begin{array}{l}\mathrm{C}-18(5 \mu \mathrm{m}, 4.6 \times 250 \mathrm{~mm}) \\
\mathrm{FD}(362-425 \mathrm{~nm}), \mathrm{R}_{\mathrm{t}^{-}}\end{array}$ & $\begin{array}{l}\text { Water:acetonitril: } \\
\text { methanol } \\
(6: 2: 3), 1 \mathrm{~mL} / \mathrm{min}\end{array}$ & $\begin{array}{l}0.02 /- \\
0.02 /-\quad(\mu \mathrm{g} / \mathrm{Kg}) \\
0.02 /- \\
0.02 /-\end{array}$ & $\begin{array}{l}\text { Bircan } \\
(2009)\end{array}$ \\
\hline $\begin{array}{l}\text { AFB1, } \\
\text { AFB2, } \\
\text { AFG1, } \\
\text { AFG2 }\end{array}$ & Noodles & $\begin{array}{l}\text { methanol:ace } \\
\text { tonitril } \\
\text { :Water } \\
(51: 9: 40)\end{array}$ & $\begin{array}{l}\text { ODS2-Spherisorb }(5 \mu \mathrm{m}, \\
4.6 \times 250 \mathrm{~mm}) \text { (capital HPLC } \\
\text { Ltd) } \\
\text { FD-, } \mathrm{R}_{\mathrm{t}^{-}}\end{array}$ & $\begin{array}{l}\text { Water:methanol:ac } \\
\text { etonitril } \\
(65: 25: 10) \\
1 \mathrm{~mL} / \mathrm{min}\end{array}$ & $\begin{array}{l}0.01-1 / 0.05-1.8 \\
0.01-1 / 0.05-1.8 \\
(\mu \mathrm{g} / \mathrm{Kg}) \\
0.01-1 / 0.05-1.8 \\
0.01-1 / 0.05-1.8\end{array}$ & $\begin{array}{l}\text { Sirhan et al. } \\
\text { (2011) }\end{array}$ \\
\hline $\begin{array}{l}\text { AFB1, } \\
\text { AFB2, } \\
\text { AFG1, } \\
\text { AFG2 }\end{array}$ & $\begin{array}{l}\text { Air in } \\
\text { poultry } \\
\text { forms }\end{array}$ & $\begin{array}{l}\text { Water } \\
(25 \mathrm{ml})+.04 \mathrm{~g} / \\
\mathrm{mL} \mathrm{NaCl}\end{array}$ & $\begin{array}{l}\text { Nova-Pak C-18 and } \\
\text { Symmetry shield TM RP-18 } \\
\text { FD-, Rt- }\end{array}$ & $\begin{array}{l}\text { Methanol+Acetonit } \\
\text { ril+ } \\
1 \% \text { phosphoric } \\
\text { acid, } 1 \mathrm{~mL} / \mathrm{min}\end{array}$ & $\begin{array}{l}0.25 /- \\
0.25 /-\mathrm{ng} / \mathrm{L} \\
0.25 /- \\
0.25 /-\end{array}$ & $\begin{array}{l}\text { Wang et al. } \\
(2008)\end{array}$ \\
\hline
\end{tabular}


Table 4: continue...

\begin{tabular}{|c|c|c|c|c|c|c|}
\hline AFB1 & Grapes & $\begin{array}{l}\text { n-hexane } \\
\text { chloroform: } \\
\text { water } \\
(40: 20)\end{array}$ & $\begin{array}{l}\text { Uptisphere C18-ODB }(5 \mu \mathrm{m}, \\
4.6 \times 250 \mathrm{~mm}) \\
\text { FD }(364-440 \mathrm{~nm})\end{array}$ & $\begin{array}{l}\text { A(1\%Phosphoric } \\
\text { acid) } \\
\text { B(Methanol:water } \\
=50: 50) \\
\text { A:B(56:44), } \\
1 \mathrm{~mL} / \mathrm{min}\end{array}$ & $0.01 /-\mu \mathrm{g} / \mathrm{Kg}$ & $\begin{array}{l}\text { El Khoury et } \\
\text { al. (2008) }\end{array}$ \\
\hline AFB1 & $\begin{array}{l}\text { Black } \\
\text { and } \\
\text { Green }\end{array}$ & $\begin{array}{l}\text { Methanol: } \\
\text { water (80:20 }\end{array}$ & $\begin{array}{l}\mathrm{C}_{18} \text { Nova-Pak }(4 \mu \mathrm{m}, \\
4.6 \times 250 \mathrm{~mm})(\text { Waters, } \\
\text { Millipore; Milford, MA) }\end{array}$ & $\begin{array}{l}\text { Water:acetonitril: } \\
\text { methanol }(20: 4: 3) \text {, } \\
1 \mathrm{~mL} / \mathrm{min}\end{array}$ & $0.15 /-\mathrm{ng} / \mathrm{g}$ & $\begin{array}{l}\text { Ghitakou et } \\
\text { al. (2006) }\end{array}$ \\
\hline
\end{tabular}

$\mathrm{AFB}_{1}$ epoxide also led to coagulopathy in animals by disrupting the formation of vitamin $\mathrm{K}$ and related clotting factors (Bababunmi et al., 1997).

\section{Toxicokinetics of AFTs}

The in vivo toxicokinetics of AFTs comprise of absorption into the bloodstream, distribution in body tissues and fluids, biotransformation (predominantly through hepatic metabolism) and elimination from the body (mainly via biliary or renal excretion). Dietary exposure to AFTs is followed by the ingestion, intestinal absorption and distribution to various body organs particularly the liver (Dhama et al., 2007). Generally, the hepatic metabolism of $\mathrm{AFB}_{1}$ occurs through demethylation, epoxidation, hydration and hydroxylation catalyzed by microsomal cytochrome P450 (CYP) monooxygenases. The major human CYP enzymes responsible for the metabolism of AFTs are CYP1 $\mathrm{A}_{2}$ which catalyzes the synthesis of $\mathrm{AFB}_{1}$-endo-8, 9-epoxide and $\mathrm{AFM}_{1}$, and CYP3A $\mathrm{A}_{4}$ which results in the formation of $\mathrm{AFB}_{1}$ exo-8, 9-epoxide and $\mathrm{AFQ}_{1}$ (Ueng et al., 1995; Gallagher et al., 1996). The o-demethylation and epoxidation (at 2, 3 double bond) of $\mathrm{AFB}_{1}$ yields $\mathrm{AFP}_{1}$ (relatively non-toxic) and $\mathrm{AFB}_{1}-8$, 9-epoxide (acutely toxic, mutagenic, and carcinogenic) respectively. $\mathrm{AFB}_{1}-8$, 9-epoxide and $\mathrm{AFM}_{1}$ undergo detoxification via glutathione conjugation catalyzed by glutathione-S-transferase enzyme in mammalian tissues (Massey et al., 1995). The hydrolysis of $\mathrm{AFB}_{1}-8$, 9-epoxide to dihydrodiol constitutes another way of its detoxification (Longouet et al., 1998). Certain avian species carry out the metabolism of $\mathrm{AFB}_{1}$ through hydration at $\mathrm{C}_{2}-\mathrm{C}_{3}$ double bond to form a relatively non-toxic metabolite referred to as $\mathrm{AFB}_{2 \mathrm{a}}$ (Patterson and Roberts, 1970). When subjected to hydroxylation at $\mathrm{C}_{4}$ or $\mathrm{C}_{22}$, the $\mathrm{AFB}_{1}$ is converted to $\mathrm{AFM}_{1}$ (acutely toxic) and $\mathrm{AFQ}_{1}$ (relatively non-toxic) respectively. Alternatively, the ketoreduction of $\mathrm{AFB}_{1}$ and $\mathrm{AFB}_{2}$ leads to aflatoxicol and dihydroaflatoxicol respectively in birds (Verma, 2005). Factors like age, breed, gender, species and physiological status have been reported to influence the in vivo disposition pattern of xenobiotics (Adil et al., 2013). For instance, ducklings, guinea-pigs and rabbits rapidly metabolize an $\mathrm{LD}_{50}$ dose of AFTs within 12 minutes. Whereas mice, sheep, pigs and chicken have moderate biotransformation capacity (an LD50 dose is metabolized in a few hours), while rats exhibit slow metabolizing potential and hence require several days to carry out the metabolism of an $\mathrm{LD}_{50}$ dose of AFTs (Patterson, 1973). The excretion of unchanged $\mathrm{AFB}_{1}$ or its metabolites occurs in urine, feces, and milk (Allcroft et al., 1968). Wong and Hsieh (1980) recorded the elimination of $54 \%, 47 \%$ and $32 \%$ of administered dose of $\mathrm{AFB}_{1}$ by mouse, rat and monkey respectively.

\section{Toxicological effects of AFTs}

\section{A) Human aflatoxicosis}

Human exposure to AFTs occurs either from the direct ingestion of contaminated nuts and maize or the carry-over of $\mathrm{AFM}_{1}$ from AFT-infected feed to milk and milk products. The clinical manifestations of human AFT poisoning vary with age, gender, nutritional status and concomitant exposure to viral or parasitic infections. Human aflatoxicosis is clinically characterized by abdominal pain, vomiting, pulmonary edema and hepatic necrosis with fatty infiltration. Various cases of acute human aflatoxicosis have been documented in developing countries (Shank et al., 1971). Outbreaks of acute toxic hepatitis have been reported in humans in Africa and China with mortality rates ranging from $10-60 \%$ (Bhat and Krishnamachari, 1977). The consumption of molded corn by households resulted in aflatoxin poisoning with at least 97 fatalities in India (Krishnamachari et al., 1975; Bhat and Krishnamachari 1977). AFT-contaminated maize had been associated with incidents of human aflatoxicosis in Kenya in 1980s and 2004 with $20 \%$ and 125 deaths respectively (Ngindu et al., 1982). Lye et al., (1995) reported acute hepatic encephalopathy in Malaysian children following the utilization of AFT-infected noodles. Likewise, chronic dietary exposure to AFTs has been implicated to cause about 250000 annual deaths resulting from hepatocellular carcinomas in exposed population of SubSaharan Africa and China (Groopman et al., 1992; Wild et al., 1992). Several studies have revealed a causal association 
between dietary AFT exposure and human hepatocellular carcinomas (Peers and Linsell, 1977; Van Rensburg et al., 1985; Lunn et al., 1997), Reye's syndrome and Kwashiorkor disease (Becroft and Webster, 1972), and childhood growth impairment (Gong et al., 2002; Okoth and Ohingo 2004; Turner et al., 2007; Sadeghi et al., 2009; Mahdavi et al., 2010; Shuaib et al., 2010).

\section{B) Aflatoxicosis in animals, birds and aquaculture species}

All animal species are predisposed to aflatoxicosis but the vulnerability of individual animals depends upon the species, gender, age and nutritional status of exposed animal together with the dose of AFT and duration of exposure (Bbosa et al., 2013). Aflatoxicosis has been reported in domestic animals including cattle (McKenzie et al., 1981; Kaleibar and Helan, 2013; Umar et al., 2015), buffaloes (Akhtar et al., 2014; Aslam et al., 2014), camels (Osman et al., 2004; Al-Hizab et al., 2015), horses (Greene and Oehme 1976; Cysewski et al., 1982), sheep (Wylie and Morehouse, 1978; Suliman et al., 1987), goats (Clark et al., 1984; Miller et al., 1984), dogs (Armbrecht et al., 1971; Arnot et al., 2012) and pigs (Yalagod Shivasharanappa et al., 2013; Olinda et al., 2016). Cattle calves, piglets and pregnant sows are highly sensitive to acute aflatoxicosis whereas sheep, goats and adult cattle are relatively resistant to acute AFT poisoning but chronic dietary exposure results in detrimental health effects and production deficits (Dhama et al., 2007). Suckling animals may be exposed to $\mathrm{AFM}_{1}$ released in the milk (Jones et al., 1994). The carry-over of $\mathrm{AFM}_{1}$ from AFT-contaminated feed poses a serious public health hazard via the consumption of contaminated milk and dairy products (Veldman et al., 1992). The reported values of median lethal dose/concentration (LD $\mathrm{L}_{50} / \mathrm{LC}_{50}$ values) of AFTs for different species of animals, birds and aquaculture have been enlisted in table-1.

Likewise, the AFT-induced pathological effects have been described in some wild animals such as deer (Quist et al., 1997), and Rhesus monkeys (Madhavan et al., 1965). Aflatoxicosis has been successfully induced in experimental animals including mice (Kanbur et al., 2011), rats (Salmon and Newberne, 1963; Wei et al., 2014), rabbits (Abd ElMageed, 1987; Abd El-Hamid, 1990; Ibrahim, 2000), guinea pigs (Butler, 1966), frogs (Grassi et al., 2007) and chinchillas (Pereyra et al., 2008). Mice were comparatively resistant to aflatoxicosis while rats were highly susceptible (Ramsdell and Eaton 1990). The general toxicological effects of aflatoxicosis in animals encompass inappetance, depression, abdominal pain, vomiting, diarrhea, pulmonary edema, ascitis, poor weight gain, reduced productivity, hepato-renal dysfunctions, convulsions, circling, blindness, photosensitization, immunosuppression, carcinogenicity, teratogenicity, abortion, hepatoencephalopathy and death following hydrocephalus and fatty infiltration of heart, liver and kidneys (Pier, 1992; Agag, 2004; Fapohunda et al., 2007; Bbosa et al., 2013). The clinicopathological effects of AFTs recorded in different animal species have been summarized in table-2.

Aflatoxicosis results in huge economic losses to poultry industry by affecting many avian species like broilers (Kubena et al., 1990; Raju and Devegowda, 2000; Aravind et al., 2003; Gowda et al., 2008), laying hens (Iqbal et al., 1983; Pandey and Chauhan, 2007), quails (Johri et al., 1990), turkeys (Quist et al., 2000), ducks (Robinson et al., 1982) and geese (Robinson et al., 1982). Ducks and turkeys were highly susceptible to AFT poisoning followed by quails while chickens were quite resistant to aflatoxicosis (Rawal et al., 2010; Monson et al., 2015). In birds, anorexia, weight loss, reduced egg production, poor pigmentation, immunosuppression, poor reproductive performance, hemato-biochemical and hormonal perturbations, hepatic necrosis with fatty infiltration and congestion, renal dysfunction, bruising and increased mortality were the most common adverse effects of AFT poisoning (Agag, 2004; Hussain et al., 2008). The clinicopathological effects of aflatoxicosis recorded in different avian species have been summarized in table-3.

Certain aquaculture species like catfish (Jantrarotai and Lovell, 1990), Indian common carp (Murjani, 2003), tilapia (Chivez-Sanchez et al., 1994; Tuan et al., 2002), trout (Lovell, 1989), sturgeon (Sepahdari et al., 2010) and shrimps (Lightner et al., 1982; Wiseman et al., 1982) are also prone to aflatoxicosis. Fry were highly sensitive than adult fish (Royes and Yanong, 2002) and Rainbow trout were more vulnerable to aflatoxicosis than channel catfish, coho salmon and zebrafish (Jantrarotai and Lovell 1990; Hendricks 1994; Dirican, 2015). Poor weight gain, retarded growth, impaired blood clotting, paleness of gills, liver tumors and increased mortality have been documented in fish affected by aflatoxicosis (Royes and Yanong, 2002). The clinicopathological effects of AFTs recorded in different aquaculture species have been summarized in table-4.

\section{C) Co-occurrence and combined toxicity of AFTs with other mycotoxins}

As humans and animals are concurrently exposed to multiple mycotoxins (Schothorst and van Egmond, 2004; Streit et al., 2013), the currently applicable single mycotoxin risk assessment strategy also requires modification. Concomitant exposure to different toxins may lead to additive, synergistic or antagonistic effects. Several techniques including the arithmetic definition of additivity, factorial design and theoretical biology-based definition of additivity have been employed to investigate the phenomenon of concurrent mycotoxicosis (Alassane-Kpembi et al., 2016).

\section{D) Natural co-occurrence of AFTs with other mycotoxins in food and feed commodities}

The natural co-existence of AFTs with other mycotoxins in food/feed commodities has been extensively studied. Rodrigues and Naehrer 2012 investigated the worldwide 
occurrence of mycotoxins in feed and foodstuff for three years and documented that $48 \%$ of analyzed samples contained two or more mycotoxins (aflatoxins, deoxynivalenol, fumonisins, ochratoxin A and zearalenone). Streit et al., 2012 reported that $75-100 \%$ of examined animal feeds contained more than a single mycotoxin. Likewise, 95\% samples of Spanish barley were contaminated with more than two mycotoxins (Ibanez-Vea et al., 2012).

The co-existance of AFTs and fumonisins has been widely reported in maize from several countries including Argentina (Broggi et al., 2007; Garrido et al., 2012), Brazil (Moreno et al., 2009), Burkina Faso (Warth et al., 2012), Cameroon (Njumbe Ediage et al., 2014), China (Sun et al., 2011), Cote D'Ivoire (Sangare-Tigori et al., 2006), Croatia (Klaric et al., 2009), Egypt (Madbouly et al., 2012), Ghana (Kpodo et al., 2000), India (Shetty and Bhat, 1997), Italy (Covarelli et al., 2011), Mozambique (Warth et al., 2012), Serbia (Krnjaja et al., 2013), South Africa (Chilaka et al., 2012), Tanzania (Kimanya et al., 2008), Turkey (Oruc et al., 2006), USA (Chamberlain et al., 1993) and Vietnam (Trung et al., 2008). Furthermore, AF and citrinin have also been concurrently identified in different food and feed ingredients (Garon et al., 2006; Richard et al., 2009).

\section{E) Simultaneous natural exposure of humans to multiple fungal toxins}

Concomitant human exposure to different mycotoxins has been evinced from several countries. Klaric et al., 2009 detected the co-existence of $\mathrm{AFB}_{1}$, ochratoxin $\mathrm{A}$ and $\mathrm{FB}_{1}$ in $20 \%$ of feed and cereal samples randomly collected from households in Croatia. Likewise, more than $40 \%$ of the pregnant Egyptian women exhibited the co-occurrence of AFT and deoxynivalenol (Piekkola et al., 2012). Abia et al., 2013 documented the concurrent occurrence of $\mathrm{AFM}_{1}$, ochratoxin A and deoxynivalenol in $63 \%$ of human urine samples in Cameroon. Similarly, more than $80 \%$ of the examined children were recorded as positive for urinary $\mathrm{FB}_{1}$ and blood AFB1-albumin adducts in Tanzania (Shirima et al., 2013).

\section{F) Toxicological interaction of AFTs with other mycotoxins in experimental animals}

Most experimental studies pertaining to toxicological interactions of AFTs with other mycotoxins have focused the simultaneous administration of $\mathrm{AFB}_{1}$ and (fumonisin $\mathrm{B}_{1}$ ) $\mathrm{FB}_{1}$. Co-exposure to $\mathrm{AFB}_{1}$ and $\mathrm{FB}_{1}$ instigated synergistic toxic effects in terms of increased carcinogenic potency of $\mathrm{FB}_{1}$ in male Fischer rats and impaired hepato-renal functions in rabbits respectively (Gelderblom et al., 2002; Orsi et al., 2007). The combination of $\mathrm{AFB}_{1}$ and $\mathrm{FB}_{1}$ was more effective in decreasing the mitogenic effect of mononuclear cells (Theumer et al., 2003), and lowering the oxidative stress markers in murine primary hepatocytes and spleen mononuclear cells (Ribeiro et al., 2010; Theumer et al., 2010) than the individual mycotoxins. Mckean et al., (2006a) observed synergistic and additive toxic effects following the co-administration of $\mathrm{AFB}_{1}$ and $\mathrm{FB}_{1}$ in male Fischer $\mathrm{F} 344$ rats and human bronchiolar epithelial cells respectively. Wistar rats manifested various histopathological lesions in hepatic, intestinal and pulmonary tissues following simultaneous exposure to $\mathrm{AFB}_{1}$ and $\mathrm{FB}_{1}$ (Theumer et al., 2008). Isolated cells from female Balb/c mice co-exposed to $\mathrm{AFB}_{1}$ and $\mathrm{FB}_{1}$ revealed enhanced level of reactive oxygen species (Abbes et al., 2016). Conversely, the human hepatoma cells $\left(\mathrm{HepG}_{2}\right)$ cotreated with $\mathrm{AFB}_{1}$ and $\mathrm{FB}_{1}$ manifested antagonistic cytotoxicity (McKean et al., 2006b). Although Friedman et al., (1997) documented the lack of toxicological interaction in rat hepatocytes culture subjected to combination of $\mathrm{AFB}_{1}$ and $\mathrm{AFB}_{2}$, the ovarian cancer cells and lung fibroblasts of human origin displayed additive effect while human umbilical vein endothelial cells exhibited synergistic effect upon coexposure to $\mathrm{AFB}_{1}$ and $\mathrm{AFB}_{2}$ (Braicu et al., 2010). Bacterial bioassays demonstrated enhanced genotoxic (Yates et al., 1987) and mutagenic (Vilar et al., 2003) effects of $\mathrm{AFB}_{1}$ resulting from the concomitant use of cyclopiazonic acid. Golli-Bennour et al. (2010) suggested an additive effect following the co-exposure of Vero cells to $\mathrm{AFB}_{1}$ and ochratoxin A. Moreover, ochratoxin A, deoxynivalenol and T2 toxin have also been reported to augment the mutagenic effect of AFB 1 (Sedmikova et al., 2001; Smerak et al., 2001). Conversely, the comet assay revealed ochratoxin A-mediated decline in DNA damage attributed to $\mathrm{AFB}_{1}$ (Corcuera et al., 2011).

\section{Detection and exposure assessment of AFTs}

The assessment of dietary exposure to AFTs entails measuring the contamination level in the food sample along with food intake surveys (Gong et al., 2016). However, the accurate estimation of contamination level can be precluded by the uneven distribution pattern of AFTs in the food and inadequate sampling technique. Biomarkers represent the crucial indicators of exposure and determinants of bioavailable dose of AFTs (Gong et al., 2016). Several molecular bio-indicators including urinary $A_{F B}-\mathrm{N}_{7}$-guanine, serum $\mathrm{AFB}_{1}$-albumin adducts and urinary $\mathrm{AFM}_{1}$ have been developed for AFT exposure estimation (Wang et al., 2001). Sputum, nasal secretions and tissue biopsies from the liver, brain and lungs of AFT-exposed individuals also reflected AFTs (Hooper et al., 2009). Furthermore, unabsorbed $\mathrm{AFB}_{1}$ or its metabolic derivatives can be detected in fecal samples collected from exposed individuals. Although, the assessment of AFTs exposure is primarily based upon the detection and quantification of AFTs or its metabolic products (e.g., AFM 1 ) in biological fluids like blood, urine, saliva and milk (Makarananda et al., 1995; Wild et al., 1998) (Table 4). Nevertheless, the characteristic short half-life of $\mathrm{AFM}_{1}$ and wide daily variation in its urinary levels limit its reliability as an effective marker of chronic exposure to AFTs (Groopman et al., 1993; Makarananda et al., 1995; Wild et al., 1998). Conversely, the AFT-albumin adduct with a longer half-life of 
30-60 days, relative stability and minimum variability can be estimated in peripheral blood to measure the long-term AFT exposure (Williams et al., 2004). Results revealed that various factors are responsible for AFTs production and all living organisms are vulnerable for its toxic effects (Asare Bediako et al., 2019; Blankson et al., 2019; Chen et al., 2019; Cheng et al., 2019; Díaz Nieto et al., 2019; Frisvad et al., 2019; Gummadidala et al., 2019; Hamza et al., 2019; Magzoub et al., 2019; Mwakinyali et al., 2019; Shahbazi and Shavisi, 2019; Singh and Cotty, 2019; Tsounidi et al., 2019; Wang et al., 2019a; Wang et al., 2019b; Wu et al., 2019; Xie et al., 2019a; Xie et al., 2019b; Yu et al., 2019; Zeng et al., 2019; Zhao et al., 2019; Zhou et al., 2019). So far, there is need to develop the detoxification methods to save food and feed items without harming the nutritional and sensory characteristics.

\section{Economic impact of AFTs}

AFT-contaminated food commodities offer a serious health hazard to more than 5 billion people across the world (Strosnider et al., 2006) and AFT exposures are usually more common in Asian and sub-Saharan African countries (Liu and $\mathrm{Wu}, 2010$ ). In developed countries, the AFT-related economic losses are primarily attributed to regulatory disposal and diminished market price of contaminated foodstuffs $\mathrm{CWu}$ and Guclu, 2012). Besides the aforementioned costs, huge losses have been ascribed to human and animal health problems, rejection of contaminated livestock, poultry and aquaculture products, research projects and regulatory interventions in developing countries (Zain, 2011; Udomkun et al., 2017). AFT contamination of corn occurring in eight Southeastern states of USA during 1980 and its subsequent consumption at hog farms led to economic losses of 97 million and 100 million US dollars, respectively (Shane, 1994). The market livestock and poultry losses were estimated to cost about 1 billion US dollars in three neighboring countries-the Philippines, Indonesia and Thailand (Lubulwa and Davis, 1994). In USA, the annual AFT-related costs owing to animal health effects and losses to peanuts and maize crops were around $\$ 500$ million (Vardon et al., 2003) whereas additional costs of nearly \$20-50 million per annum were required to overcome the problem of AFT contamination (Robens and Cardwell, 2003). Moreover, the US maize growers encountered an estimated loss of $\$ 163$ million per annum on account of aflatoxin issue $(\mathrm{Wu}, 2006)$.

\section{Regulation of AFTs in food and feed items}

Appropriate regulatory and legislative measures are requisite to overcome the aflatoxin contamination of food and feed commodities for increasing the market value of products derived from plants and animals and reducing the healthcare costs. Although, more than 100 nations have established the maximum admissible levels of AFTs in food or feed items (Wu and Guclu, 2012), improved detection facilities and optimal legislative practices are still lacking in several developing countries, predominantly the sub-Saharan
African nations (Udomkun et al., 2017). Such regulations typically reveal the strictest levels of AFTs for export and human consumption products while lowest levels are meant for items of industrial usage (Udomkun et al., 2017). Potential intervention approaches like coordination of supply chain, technical capacity building, provision of suitable incentives to control fungal infections and enhanced public awareness through extension services are vital to address the issue of aflatoxicosis (Deng et al., 2018; Fan et al., 2018; Klingelhöfer et al., 2018; Moon et al., 2018; Peng et al., 2018; Salem et al., 2018; Spanjer, 2019; Vidal et al., 2018; Xu et al., 2018).

\section{CONCLUSION AND RECOMMENDATIONS}

AFTs represent toxic fungal metabolites implicated to provoke considerable economic losses and potential deleterious effects on human as well as animal health. Food commodities such as cereals, fruits, nuts, spices, oil seeds, beans and dried peas are primarily affected by AFTs. Various effective control measures in terms of biological, chemical, physical and genetic engineering techniques have been applied for the alleviation and control of AFTs in the food. The high levels of AFTs in food items and associated illeffects are of greater concern in developing countries with temperate and tropical climate, food scarcity and lack of proper control strategies. The accessibility of diverse foods together with the implementation of appropriate regulatory policies can help to curtail the AFT contamination at least in developed countries. Furthermore, understanding the molecular and genetic basis of AFT biosynthesis, improved management procedures, better allocation of monitoring efforts, and adjustment of agronomic practices are requisite to circumvent AFT contamination.

\section{REFERENCES}

Abbes, S., Ben Salah-Abbès, J., Jebali, R., Younes, R.B., Oueslati, R., 2016. Interaction of aflatoxin B1 and fumonisin B1 in mice causes immunotoxicity and oxidative stress: Possible protective role using lactic acid bacteria. Journal of Immunotoxicology 13(1), 46-54.

Abd El-Hamid, A.M., 1990. Occurrence of some mycotoxins (aflatoxins, ochratoxin A, citrinin, zearaltenone and vomitoxin) in various Egyptian feeds. Archives of Animal Nutrition 40, 647-664.

Abia, W.A., Warth, B., Sulyok, M., Krska, R., Tchana, A., Njobeh, P.B., Turner, P.C., Kouanfack, C., Eyongetah, M., Dutton, M., Moundipa, P.F., 2013. Bio-monitoring of mycotoxin exposure in Cameroon using a urinary multi-biomarker approach. Food and Chemical Toxicology 62, 927-934.

Adil, M., Sikandar, A., Waheed, U., Idrees, M., 2013. A rational pharmacotherapeutic approach for veterinary practitioners. International Journal of Veterinary Science 2(1), 12-16. 
Agag, B.I., 2004. Mycotoxins in foods and feeds: Aflatoxins. Association of Universal Bullettin of Environmental Research 7(1), 173-191.

Akhtar, R., Sardar, M., Saima, N., Saleem, G., Imran, S., Aslam, A., 2014. Responses of Nili-Ravi buffalo to aflatoxin B1 with and without toxin adsorbents. Journal of Animal and Feed Sciences 23(4), 317-323.

Alassane-Kpembi, I., Schatzmayr, G., Taranu, I., Marin, D., Puel, O., Oswald, I.P., 2017. Mycotoxins co-contamination: Methodological aspects and biological relevance of combined toxicity studies. Critical Reviews in Food Science and Nutrition 57(16), 3489-3507.

Al-Hizab, F.A., Al-Gabri, N.A.M., and Barakat, S.E.M., 2015. Effect of Aflatoxin B1 (AFB1) residues on the pathology of camel liver. Asian Journal of Animal and Veterinary Advances 10(4), 173-178.

Allcroft, R., Roberts, B.A., Lloyd, M.K., 1968. Excretion of aflatoxin in a lactating cow. Food and Cosmetics Toxicology 6, 619- 625 .

Angle, J.S., 1986. Aflatoxin decomposition in various soils. Journal of Environmental Science and Health B 21(4), 277-288.

Aravind, K.L., Patil, V.S., Devegowda, G., Umakantha, B., Ganpule, S.P., 2003. Efficacy of esterified glucomannan to counteract mycotoxicosis in naturally contaminated feed on performance and serum biochemical and hematological parameters in broilers. Poultry Science 82(4), 571-576.

Armbrecht, B.H., Geleta, J.N., Shalkop, W.T., 1971. A subacute exposure of beagle dogs to aflatoxin. Toxicology and Applied Pharmacology 18(3), 579-585.

Arnot, L.F., Duncan, N.M., Coetzer, H., Botha, C.J., 2012. An outbreak of canine aflatoxicosis in Gauteng Province, South Africa. Journal of the South African Veterinary Association 83(1), 01-04.

Asare Bediako, K., Ofori, K., Offei, S.K., Dzidzienyo, D., Asibuo, J.Y., Adu Amoah, R., 2019. Aflatoxin contamination of groundnut (Arachis hypogaea L.): Predisposing factors and management interventions. Food Control 98, 61-67.

Aslam, N., Iqbal, Z.M., Warriach, H.M., and Wynn, P.C., 2014. Pattern of partitioning of aflatoxins from feed to urine and its effect on serum chemistry in Nili-Ravi buffalo heifers. Animal Production Science 54(10), 1671-1675.

Bababunmi, E.A., Thabrew, I., Bassir, O., 1997. Aflatoxin induced coagulopathy in different nutritionally classified animal species. World Review of Nutrition and Dietetics 34, 161-181.

Bailey, G.S., 1994. Role of aflatoxin-DNA adducts in the cancer process. In: Eaton, D.L., Groopman, J.D. (Eds.), The Toxicology of Aflatoxins: Human Health, Veterinary, and Agricultural Significance. Academic Press, San Diego, CA, p. 137-148.

Bankole, S., Schollenberger, M., Drochner, W., 2006. Mycotoxins in food systems in Sub Saharan Africa: A review. Mycotoxin Research, 22(3), 163-169.

Bbosa, G.S., Kitya, D., Lubega, A., Ogwal-Okeng, J., Anokbonggo, W.W., Kyegombe, D.B., 2013. Review of the biological and health effects of aflatoxins on bodyorgans and body systems, in Aflatoxins - Recent Advances and Future Prospects, ed. M. Razzaghi-Abyaneh (Rijeka: InTech), p. 239-265.

Becroft, D.M.O., Webster, D.R., 1972. Aflatoxins and Reye's disease. British Medical Journal 4, 117.

Bennett, J.W., Papa, K.E., 1988. The aflatoxigenic A. Spp. Advances in Plant Pathology: Genetics of Plant Pathogenic Fungi 6, 263-80.

Bhat, R.V., Krishnamachari, K.A.V.R., 1977. Follow-up study of aflatoxic hepatitis in parts of Western India. Indian Journal of Medical Research 66, 55-58.

Blankson, G.K., Mills-Robertson, F.C., Ofosu, I.W., 2019. Survey of occurrence levels of Aflatoxins in selected locally processed cereal-based foods for human consumption from Ghana. Food Control 95, 170-175.

Bock, C.H., Cotty, P.J., 1999. The relationship of gin date to aflatoxin contamination of cottonseed in Arizona. Plant Disease 83, 279-285.

Bock, C.H., Mackey, B., Cotty, P.J., 2004. Population dynamics of $A$. flavus in the air of an intensively cultivated region of south-west Arizona. Plant Pathology 53, 422-433.

Bok, J.W., Keller, N.P., 2004. LaeA, a regulator of secondary metabolism in A. Spp. Eukaryotic cell 3(2), 527-535.

Bonsi, P., Agusti-Tocco, G., Palmery, M., Giorgi, M., 1999. Aflatoxin B1 is an inhibitor of cyclic nucleotide phosphodiesterase activity. General Pharmacology 32, 615-619.

Bowen, K.L., Mack, T.P., 1993. Relationship of Damage from the Lesser Cornstalk Borer to A. flavus Contamination in Peanuts 2. Journal of Entomological Science 28(1), 29-42.

Braicu, C., Berindan-Neagoe, I.O.A.N.A., Chedea, V.S., Balacescu, L., Brie, I., Soritau, O.,Socaciu, C., Irimie, A., 2010. Individual and combined cytotoxic effects of the major four aflatoxins in different in vitro stabilized systems. Journal of Food Biochemistry 34(5), 1079-1090.

Broggi, L. E., Pacin, A. M., Gasparovic, A., Sacchi, C., Rothermel, A., Gallay, A., and Resnik, S., 2007. Natural occurrence of aflatoxins, deoxynivalenol, fumonisins and zearalenone in maize from Entre Rios Province, Argentina. Mycotoxin Research 23(2), 59-64.

Buchanan, R.L., Lewis, D.F., 1984. Regulation of aflatoxin biosynthesis: effect of glucose on activities of various glycolytic enzymes.Applied and Environmental Microbiology 48(2), 306-310.

Butler, W.H., 1996. Acute Toxicity of Aflatoxin B in Guinea Pigs. Journal of Pathology and Bacteriology 91, 277-280.

Calvo, A.M., Bok, J., Brooks, W., Keller, N.P., 2004. veA required for toxin and sclerotial production in $A$. parasiticus. Applied and Environmental Microbiology 70(8), 4733-4739.

Cary, J.W., Montalbano, B.G., Ehrlich, K.C., 2000. Promoter elements involved in the expression of the $A$. parasiticus aflatoxin biosynthesis pathway gene avnA. Biochimica et Biophysica Acta (BBA)-General Structure and Expression 1491(1), 7-12. 
Chamberlain, W.J., Bacon, C.W., Norred, W.P., Voss, K.A., 1993. Levels of fumonisin B1 in corn naturally contaminated with aflatoxins. Food and Chemical Toxicology 31, 995998.

Chauhan, Y.S., Wright, G.C, Rachaputi, N,C., 2008. Modelling climatic risks of aflatoxin contamination in maize. Australian Journal of Experimental Agriculture 48, 358366.

Chauhan, Y.S., Wright, G.C., Rachaputi, R.C.N., 2010. Application of a model to assess aflatoxin risk in peanuts. Journal of Agricultural Science 148, 341-51.

Chavez-Sanchez, M.C., Palacios, C.M., Moreno, I.O., 1994. Pathological effects of feeding young Oreochromis niloticus diets supplemented with different levels of aflatoxin B1. Aquaculture 127(1), 49-60.

Chen, Y., Kong, Q., Liang, Y., 2019. Three newly identified peptides from Bacillus megaterium strongly inhibit the growth and aflatoxin B1 production of Aspergillus flavus. Food Control 95, 41-49.

Cheng, X., Vella, A., Stasiewicz, M.J., 2019. Classification of aflatoxin contaminated single corn kernels by ultraviolet to near infrared spectroscopy. Food Control 98, 253-261.

Chilaka, C.A., De Kock, S., Phoku, J.Z., Mwanza, M., Egbuta, M. A., Dutton, M.F., 2012. Fungal and mycotoxin contamination of South African commercial maize. Journal of Food Agriculture and Environment 10, $296 \mathrm{e} 303$.

Chulze, S., Varsavsky, E., Fusero, S., Dalcero, A., Farnochi, C., 1991. Effect of the lipid fraction of sunflower seeds on aflatoxin production by $A$. parasiticus. Mycological Research 95(2), 254-256.

Clark, J.D., Hatch, R.C., Miller, D.M., Jain, A.V., 1984. Caprine aflatoxicosis: experimental disease and clinical pathologic changes. American Journal of Veterinary Research 45(6), 1132-1135.

Corcuera, L.A., Arbillaga, L., Vettorazzi, A., Azqueta, A., de Cerain, A.L., 2011. Ochratoxin A reduces aflatoxin B1 induced DNA damage detected by the comet assay in Hep G2 cells. Food and Chemical Toxicology 49, 2883-2889.

Cotty, P. J., Jaime-Garcia, R., 2007. Influences of climate on aflatoxin producing fungi and aflatoxin contamination. International Journal of Food Microbiology 119, 109115.

Cotty, P., 1988. Aflatoxin and sclerotial production by $A$. flavus: influence of $\mathrm{pH}$. Phytopathology 78(9), 12501253.

Cotty, P.J., Cardwell, K.F., 1999. Divergence of West African and North American communities of $A$. section Flavi. Applied and Environmental Microbiology 65, 2264-2266.

Covarelli, L., Beccari, G., Salvi, S., 2011. Infection by mycotoxigenic fungal species and mycotoxin contamination of maize grain in Umbria, central Italy. Food and Chemical Toxicology 49, 2365-2369.

Craufurd, P.Q., Prasad, P.V.V., Waliyar, F., Taheri, A., 2006. Drought, pod yield, pre-harvest A. infection and aflatoxin contamination on peanut in Niger. Field Crops Research 98(1), 20-29.
Cuero, R., Ouellet, T., Yu, J., Mogongwa, N., 2003. Metal ion enhancement of fungal growth, gene expression and aflatoxin synthesis in $A$. flavus: RT-PCR characterization. Journal of Applied Microbiology 94(6), 953-961.

Cysewski, S.J., Pier, A.C., Baetz, A.L., Cheville, N.F., 1982. Experimental equine aflatoxicosis. Toxicology and Applied Pharmacology 65, 354-365.

D’Mello, J.P.F., Macdonald, A.M.C., Postel, D., Dijksma, W.T.P., DuJardin, A., Placinta, C.M., 1998. Pesticide use and mycotoxin production in Fusarium and A. phytopathogens. European Journal of Plant Pathology 104, 741-751.

Deng, J., Zhao, L., Zhang, N.-Y., Karrow, N.A., Krumm, C.S., Qi, D.-S., Sun, L.-H., 2018. Aflatoxin B1 metabolism: Regulation by phase I and II metabolizing enzymes and chemoprotective agents. Mutation Research/Reviews in Mutation Research 778, 79-89.

Dhama, K., Chauhan, R. S., Mahendran, M., Singh, K. P., Telang, A. G., Singhal, L., Tomar, S., 2007. Aflatoxins-hazard to livestock and poultry production: A review. Journal of Immunology and Immunopathology 9 (1 and 2), 1-15.

Díaz Nieto, C.H., Granero, A.M., Garcia, D., Nesci, A., Barros, G., Zon, M.A., Fernández, H., 2019. Development of a thirdgeneration biosensor to determine sterigmatocystin mycotoxin: An early warning system to detect aflatoxin B1. Talanta 194, 253-258.

Dirican, S., 2015. A review of effects of aflatoxins in aquaculture. Applied Research Journal 1, 192-196.

Dorner, J.W., 2008. Management and prevention of mycotoxins in peanuts. Food Additives and Contaminants 25, 203-208.

Dorner, J.W., Cole, R.J., Connick, W.J., Daigle, D.J., Mcguire, M. R., Shasha, B.S., 2003. Evaluation of biological control formulations to reduce aflatoxin contamination in peanuts. Biological Control 26, 318-324.

Dorner, J.W., Cole, R.J., Sanders, T.H., and Blankenship, P.D., 1989. Interrelationship of kernel water activity, soil temperature, maturity, and phytoalexin production in preharvest aflatoxin contamination of drought-stressed peanuts. Mycopathologia 105, 117-128.

Ehrlich, K.C., Chang, P.-K., Scharfenstein, J.S.L., Cary, J.W., Crawford, J.M., Townsend, C.A., 2010. Absence of the aflatoxin biosynthesis gene, norA, allows accumulation of deoxyaflatoxin $\mathrm{B} 1$ in $A$. flavus cultures. FEMS Microbiology Letters 305, 65-70.

Fan, Y., Liu, L., Zhao, L., Wang, X., Wang, D., Huang, C., Zhang, J., Ji, C., Ma, Q., 2018. Influence of Bacillus subtilis ANSB060 on growth, digestive enzyme and aflatoxin residue in Yellow River carp fed diets contaminated with aflatoxin B1. Food and Chemical Toxicology 113, 108114.

Fanelli, C., Fabbri, A.A., Brasini, S., de Luca, C., Passi, S., 1995. Effect of different inhibitors of sterol biosynthesis on both fungal growth and aflatoxin production. Natural Toxins 3, 109-113. 
Fapohunda, S.O., Ezekiel, C.N., Alabi, O.A., Omole, A., Chioma, S.O., 2008. Aflatoxin-mediated sperm and blood cell abnormalities in mice fed with contaminated corn. Mycobiology 36(4), 255-259.

Flaherty, J.E., Payne, G. A., 1997. Overexpression of aflR leads to upregulation of pathway gene transcription and increased aflatoxin production in A. flavus. Applied and Environmental Microbiology 63(10), 3995-4000.

Foster, P.L., Eisenstadt, E., Miller, J.H., 1983. Base substitution mutations induced by metabolically activated aflatoxin B1. Proceedings of National Academy of Sciences 80, 2695-2698.

Friedman, L., Gaines, D.W., Chi, R.K., Smith, M.C., Braunberg, R.C., Thorpe, C.W., 1997. Interaction of aflatoxins as measured by their biochemical action on rat liver slices and hepatocytes. Toxic Substance Mechanism 16, 15-41.

Frisvad, J.C., Hubka, V., Ezekiel, C.N., Hong, S.B., Nováková, A., Chen, A.J., Arzanlou, M., Larsen, T.O., Sklenář, F., Mahakarnchanakul, W., Samson, R.A., Houbraken, J., 2019. Taxonomy of Aspergillus section Flavi and their production of aflatoxins, ochratoxins and other mycotoxins. Studies in Mycology 93, 1-63.

Gallagher, E.P., Kunze,K.L., Stapleton, P.L. and Eaton, D.L., 1996. The kinetics of aflatoxin B1 oxidation by human cDNA-expressed and human liver microsomal cytochromes P450 1A2 and 3A4. Toxicology and Applied Pharmacology 141, 595-606

Gallo, A., Solfrizzo, M., Epifani, F., Panzarini, G., and Perrone, G., 2016. Effect of temperature and water activity on gene expression and aflatoxin biosynthesis in A. flavus on almond medium. International Journal of Food Microbiology 217, 162-169.

Garon, D., Richard, E., Sage, L., Bouchart, V., Pottier, D., Lebailly, P., 2006. Mycoflora and multimycotoxin detection in corn silage: Experimental study. Journal of Agricultural and Food Chemistry 54, 3479-3484.

Garrido, C.E., Hern_andez Pezzani, C., Pacin, A., 2012. Mycotoxins occurrence in Argentina's maize (Zea mays L.), from 1999 to 2010. Food Control 25, 660e665.

Gelderblom, W.C.A., Marasas, W.F.O., Lebepe-Mazur, S., Swanevelder, S., Vessey, C.J., Hall Pde L., 2002. Interaction of fumonisin B 1 and aflatoxin B 1 in a short-term carcinogenesis model in rat liver. Toxicology 171(2), 161-173.

Golli Bennour, E. E., Bouaziz, C., Ladjimi, M., Renaud, F., Bacha, H., 2009. Comparative mechanisms of zearalenone and ochratoxin A toxicities on cultured HepG2 cells: is oxidative stress a common process? Environmental Toxicology 24, 538-548.

Gong, Y.Y., Cardwell, K., Hounsa, A., Egal, S., Turner, P.C., 2002. Dietary aflatoxin exposure and impaired growth in young children from Benin and Togo: cross sectional study. British Medical Journal 325, 20-21

Gong, Y.Y., Watson, S., Routledge, M.N., 2016. Aflatoxin exposure and associated human health effects, a review of epidemiological studies. Food Safety 4(1), 14-27.
Gowda, N.K.S., Ledoux, D.R., Rottinghaus, G.E., Bermudez, A.J., Chen, Y.C., 2008. Efficacy of turmeric (Curcuma longa), containing a known level of curcumin, and a hydrated sodium calcium aluminosilicate to ameliorate the adverse effects of aflatoxin in broiler chicks. Poultry Science 87(6), 1125-1130.

Grassi, T.F., Pires, P.W., Barbisan, L.F., Dal Pai-Silva, M., Said, R.A., de Camargo, J.L.V., 2007. Liver lesions produced by aflatoxins in Rana catesbeiana (bullfrog). Ecotoxicology and Environmental Safety 68(1), 71-78.

Greene, H.J., Oehme, F.W., 1976. A possible case of equine aflatoxicosis. Clinical Toxicology 9(2), 251-254.

Greene-McDowelle, D.M., Ingber, B., Wright, M.S., Zeringue Jr, H.J., Bhatnagar, D., Cleveland, T.E., 1999. The effects of selected cotton-leaf volatiles on growth, development and aflatoxin production of Aspergillus parasiticus. Toxicon 37, 883-893.

Groopman J.D., 1993. Molecular dosimetry methods for assessing human aflatoxin exposures. In: Eaton DL, Groopman JD, eds. The toxicology of aflatoxins: human health, veterinary and agricultural significance. London: Academic Press, pp. 259 -79.

Groopman, J.D., Zhu, J.Q., Donahue, P.R., Pikul, A., Zhang, L.S., Chen, J.S., Wogan, G.N., 1992. Molecular dosimetry of urinary aflatoxin-DNA adducts in people living in Guangxi autonomous region, People's Republic of China. Cancer Res. 52, 45-52.

Gummadidala, P.M., Omebeyinje, M.H., Burch, J.A., Chakraborty, P., Biswas, P.K., Banerjee, K., Wang, Q., Jesmin, R., Mitra, C., Moeller, P.D.R., Scott, G.I., Chanda, A., 2019. Complementary feeding may pose a risk of simultaneous exposures to aflatoxin M1 and deoxynivalenol in Indian infants and toddlers: Lessons from a mini-survey of food samples obtained from Kolkata, India. Food and Chemical Toxicology 123, 9-15.

Hamza, Z., El-Hashash, M., Aly, S., Hathout, A., Soto, E., Sabry, B., Ostroff, G., 2019. Preparation and characterization of yeast cell wall beta-glucan encapsulated humic acid nanoparticles as an enhanced aflatoxin B1 binder. Carbohydrate Polymers 203, 185-192.

Heathcoate, J.G., Hibbert, J.R., 1978. Aflatoxins: chemical and biological aspects Elsevier Applied Science, Amsterdam.

Hell, K., Cardwell, K.F., Poehling, H.M., 2003. Relationship between management practices, fungal infection and aflatoxin for stored maize in Benin. Journal of Phytopathology 151, 690-698.

Hendricks J.D., 1994. Carcinogenicity of aflatoxins in nonmammalian organisms. In: Eaton DL, Groopman JD (eds) The toxicology of aflatoxins: human health, veterinary, and agricultural significance. Academic Press, New York, pp.103-136

Hill, R.A., Blankenship, P.D., Cole, R.J., Sanders, T.H., 1983. Effect of soil moisture and temperature on preharvest invasion of peanuts by the $A$. flavus group and subsequent aflatoxin development. Applied and Environmental Microbiology 45, 628-633. 
Hooper, D.G., Bolton, V.E., Guilford, F.T., Straus, D.C., 2009. Mycotoxin detection in human samples from patients exposed to environmental molds. International Journal of Molecular Sciences 10, 1465-1475

Horn, B.W., Dorner, J.W., 1999. Regional differences in production of aflatoxin B1 and cyclopiazonic acid by soil isolates of $A$. flavus along a transect within the United States. Applied and Environmental Microbiology 65(4), 1444-1449.

Howard, D.H., 1983. Fungi pathogenic for humans and animals. Part A. Biology. Marcel Dekker, Inc., New York \& Basel.

Howarth, B., Wyatt, R.D., 1976. Effect of dietary aflatoxin on fertility, hatchability, and progeny performance of broiler breeder hens. Applied and Environmental Microbiology 31(5), 680-684.

Hussain, Z., Khan, M.Z., Hassan, Z.U., 2008. Production of aflatoxins from $A$. flavus and Acute aflatoxicosis in young broiler chicks. Pakistan Journal of Agricultural Sciences 45(1), 95-102.

Ibáñez-Vea, M., González-Peñas, E., Lizarraga, E., de Cerain, A. L., 2012. Co-occurrence of mycotoxins in Spanish barley: A statistical overview. Food Control 28(2), 295-298.

Ibrahim, K.I.K., 2000. Effect of aflatoxins and ascorbic acid on some productive and reproductive parameters in male rabbits. M.Sc. Thesis, Faculty of Agriculture, Alexendria University, Egypt.

Iqbal, Q.K., Rao, P.V., Reddy, S.J., 1983. Dose-response relationship of experimentally induced aflatoxicosis in commercial layers. Journal of Animal Science 53, 12771280.

Iqbal, S.Z., Paterson, R.R.M., Bhatti, I.A., Asi, M.R., 2011. Comparing aflatoxin contamination in chilies from Punjab, Pakistan produced in summer and winter. Mycotoxin Research 27(2), 75-80.

Jaime-Garcia, R., Cotty, P.J., 2003. Aflatoxin contamination of commercial cottonseed in South Texas. Phytopathology 93, 1190-1200.

Jantrarotai, W., Lovell, R.T., 1990. Subchronic toxicity of dietary aflatoxin B1 to channel catfish. Journal of Aquatic Animal Health 2, 248-254.

Jantrarotai, W., Lovell, R.T., Grizzle, J.M., 1990. Acute toxicity of aflatoxin B1 to channel catfish. Journal of Aquatic Animal Health 2(4), 237-247.

Jayashree, T., Subramanyam, C., 2000. Oxidative stress as a prerequisite for aflatoxin production by $A$. parasiticus. Free Radical Biology and Medicine 29,981-85

Johri, T. S., Agrawal, R.C., Sadagopan, V.R., 1990. Effect of low dietary levels of aflatoxin on laying quail (Coturnix coturnix japonica) and their response to dietary modifications. Indian Journal of Animal Sciences 60, 355359.

Jones, F.T., Beth, M., Genter, M.M., Hagler, W.M., Hansen, J.A., Mowrewy, B.A., Poore, M.H., Whitlow, L.W., 1994. Understanding and coping with effects of mycotoxins in livestock feed and forage. Electronic publication No.
DR0-29, NCCES, North Carolina State Univ., Raleigh, North Carolina.

Kachholz, T., Demain, A.L., 1983. Nitrate repression of averufin and aflatoxin biosynthesis. Journal of Natural Products 46(4), 499-506.

Kaleibar, M.T., Helan, J.A., 2013. A field outbreak of aflatoxicosis with high fatality rate in feedlot calves in Iran. Comparative Clinical Pathology 22(6), 1155-1163.

Kebede, H., Abbas, H.K., Fisher, D.K., Bellaloui, N., 2012. Relationship between aflatoxin contamination and physiological responses of corn plants under drought and heat stress. Toxins 4(11), 1385-1403.

Keller, N.P., Nesbitt, C., Sarr, B., Phillips, T.D., Burow, G.B., 1997. $\mathrm{pH}$ regulation of sterigmatocystin and aflatoxin biosynthesis in A. Spp. Phytopathology 87(6), 643-648.

Kim, J.H., Yu, J., Mahoney, N., Chan, K.L., Molyneux, R.J., Varga, J., Bhatnagar, D., Cleveland, T.E., Nierman, W.C. and Campbell, B.C., 2008. Elucidation of the functional genomics of antioxidant-based inhibition of aflatoxin biosynthesis. International Journal of Food Microbiology 122(1), 49-60.

Kimanya, M. E., De Meulenaer, B., Tiisekwa, B., NdomondoSigonda, M., Devlieghere, F., Van Camp, J., Kolsteren, P., 2008. Co-occurrence of fumonisins with aflatoxins in home-stored maize for human consumption in rural villages of Tanzania. Food Additives and Contaminants 25(11), 1353-1364.

Klaric, M.S., Cvetnic, Z., Pepeljnjak, S., Kosalec, I., 2009. Cooccurrence of aflatoxins, ochratoxin A, fumonisins, and zearalenone in cereals and feed, determined by competitive direct enzyme-linked immunosorbent assay and thin-layer chromatography. Archives of Industrial Hygiene and Toxicology 60, 427-434.

Klich, M.A., 2002. Biogeography of A. species in soil and litter. Mycologia 94, 21-27.

Klich, M.A., 2007. Environmental and developmental factors influencing aflatoxin production by $A$. flavus and $A$. parasiticus. Mycoscience 48, 71-80.

Klingelhöfer, D., Zhu, Y., Braun, M., Bendels, M.H.K., Brüggmann, D., Groneberg, D.A., 2018. Aflatoxin Publication analysis of a global health threat. Food Control 89, 280-290.

Kpodo, K., Thrane, U., Hald, B., 2000. Fusaria and fumonisins in maize from Ghana and their co-occurrence with aflatoxins. International Journal of Food Microbiology 61, 147-157.

Krishnamachari, K.A.V.R., Bhat, R. V., Nagarajan, V., Tilak, T. B.G., 1975. Hepatitis due to aflatoxicosis-An outbreak in western India. Lancet 1, 1061-1063.

Krnjaja, V., Lević, J., Stanković, S., Petrović, T., Tomić, Z., Mandić, V., Bijelić, Z., 2013. Moulds and mycotoxins in stored maize grains. Biotechnology in Animal Husbandry 29, 527-536.

Kubena, L.F., Harvey, R.B., Huff, W.E., Corrier, D.E., Phillips, T.D., Rottinghaus, G.E., 1990. Efficacy of a hydrated sodium calcium aluminosilicate to reduce the toxicity of 
aflatoxin and T-2 toxin. Poultry Science 69(7), 10781086.

Lewis, L., Onsongo, M., Njapau, H., Schurz-Rogers, H., Luber, G., Kieszak, S., Nyamongo, J., Backer, L., Dahiye, A.M., Misore, A. and DeCock, K., 2005. Aflatoxin contamination of commercial maize products during an outbreak of acute aflatoxicosis in eastern and central Kenya. Environmental Health Perspectives 113(12), 1763-1767.

Lightner, D.V., Redman, R.M., Price, R.L., Wiseman, M.O., 1982. Histopathology of aflatoxicosis in the marine shrimp Penaeus stylirostris and P. vannamei. Journal of Invertebrate Pathology 40(2), 279-291.

Lillehoj, E.B., 1991. Aflatoxin: an ecologically elicited activation signal. In: Smith, J.E., Anderson, R.A. (Eds.), Mycotoxins and Animal Foods. CRC Press, Boca Raton, FL, pp.119-139.

Liu, Y., Wu, F., 2010. Global burden of aflatoxin-induced hepatocellular carcinoma: a risk assessment. Environmental Health Perspectives 118, 818-824.

Logrieco, A., Visconti, A., 2004. An Overview on Toxigenic Fungi and Mycotoxins in Europe. Dordrecht Netherlands: Kluwer Academic Publishers.

Longouet, S., Johnson, W.W., Guillouzo, A., Guengerich, F.P., 1998. Detoxication of aflatoxin B1 as a model for carcinogen metabolism. In Vitro Molecular Toxicology 11, 95-101.

Lovell, R.T., 1989. Nutrition and feeding of fish. Van Nostrand Reinhold, New York.

Lubulwa, A.S.G. Davis, J.S., 1994. Estimating the social costs of the impacts of fungi and aflatoxins in maize and peanuts. In: Highley, E., Wright, E.J., Banks, H.J. and Champ, B.R. (eds.) Stored product protection: Proceedings of the $6^{\text {th }}$ International Working Conference on Stored-product Protection. CAB International, Wallingford, UK, pp. 10171042.

Luchese, R.H., Harrigan, W.F., 1993. Biosynthesis of aflatoxin-the role of nutritional factors. Journal of Applied Bacteriology 74(1), 5-14.

Lunn, R.M., Zhang, Y.J., Wang, L.Y., Chen, C.J., Lee, P.H., Lee, C.S., Tsai, W.Y. and Santella, R.M., 1997. P53 mutations, chronic hepatitis b virus infection, and alfatoxin exposure in hepatocellular carcinoma in Taiwan. Cancer Research 57(16), 3471-3477.

Lye, M.S., Ghazali, A.A., Mohan, J., Alwin, N., Nair, R.C., 1995. An outbreak of acute hepatic encephalopathy due to severe aflatoxicosis in Malaysia. American Journal of Tropical Medicine and Hygiene 53, 68-72.

Madbouly, A.K., Ibrahim, M.I., Sehab, A.F., Abdel-Wahhab, M.A., 2012. Co-occurrence of mycoflora, aflatoxins and fumonisins in maize and rice seeds from markets of different districts in Cairo, Egypt. Food Additives and Contaminants: Part B 5(2), 112-120.

Madhavan, T.V., Tulpule, P.G., Gopalan, C., 1965. Aflatoxininduced hepatic fibrosis in rhesus monkeys: pathological features. Archives of Pathology 79, 466-469.
Magan, N., Aldred, D., 2007. Post-harvest control strategies: minimizing mycotoxins in the food chain. International Journal of Food Microbiology 119, 131-139.

Magan, N., Hope, R., Cairns, V., Aldred, D., 2003. Post-harvest fungal ecology: impact of fungal growth and mycotoxin accumulation in stored grain. European Journal of Plant Pathology 109(7), 723-730.

Magan, N., Medina, A., Aldred, D., 2011. Possible climate change effects on mycotoxin contamination of food crops pre- and post-harvest. Plant Pathology 60,150-163.

Magzoub, R.A.M., Yassin, A.A.A., Abdel-Rahim, A.M., Gubartallah, E.A., Miskam, M., Saad, B., Sabar, S., 2019. Photocatalytic detoxification of aflatoxins in Sudanese peanut oil using immobilized titanium dioxide. Food Control 95, 206-214.

Mahdavi, R., Nikniaz, L., Arefhosseini, S.R., Vahed Jabbari, M., 2010. Determination of aflatoxinM1 in breast milk samples in Tabriz-Iran. Maternal and Child Health Journal 14, 141-45

Mahoney, N., Molyneux, R.J., 2004. Phytochemical inhibition of aflatoxigenicity in A. flavus by constituents of walnut Uuglans regia). Journal of Agricultural and Food Chemistry 52(7), 1882-1889.

Makarananda, K., Pengpan, U., Srisakulthong, M., Yoovathaworn, K., Sriwatanakul, K., 1998. Monitoring of aflatoxin exposure by biomarkers. Journal of Toxicological Sciences 23, 155-159.

Marroquín-Cardona, A.G., Johnson, N.M., Phillips, T.D., Hayes, A.W., 2014. Mycotoxins in a changing global environment-a review. Food and Chemical Toxicology, 69, 220-230.

Massey, T.E., Stewart, R.K., Daniels, J.M., Liu, L., 1995. Biochemical and molecular aspects of mammalian susceptibility to aflatoxin B1 carcinogenicity. Proceedings of the Society for Experimental Biology and Medicine 208, 213-227.

McKean, C., Tang, L., Billam, M., Tang, M., Theodorakis, C.W., Kendall, R.J., Wang, J.S., 2006a. Comparative acute and combinative toxicity of aflatoxin B1 and T-2 toxin in animals and immortalized human cell lines. Journal of Applied Toxicology 26, 139-147.

McKean, C., Tang, L., Tang, M., Billam, M., Wang, Z., Theodorakis, C.W., Kendall, R.J. Wang, J.S., 2006b. Comparative acute and combinative toxicity of aflatoxin $\mathrm{B}_{1}$ and fumonisin $\mathrm{B}_{1}$ in animals and human cells. Food and Chemical Toxicology 44(6), 868-876.

McKenzie, R.A., Blaney, B.J., Connole, M.D., Fitzpatrick, L.A., 1981. Acute aflatoxicosis in calves fed peanut hay. Australian Veterinary Journal 57(6), 284-286.

Meyers, D. M., Obrian, G., Du, W. L., Bhatnagar, D., Payne, G. A. (1998). Characterization of aflJ, a gene required for conversion of pathway intermediates to aflatoxin. Applied and Environmental Microbiology 64(10), 3713-3717.

Miller, D.M., Clark, J.D., Hatch, R.C., Jain, A.V., 1984. Caprine aflatoxicosis: serum electrophoresis and pathologic 
changes. American Journal of Veterinary Research 45(6), 1136-1141.

Minto, R.E., Townsend, C.A., 1997. Enzymology and molecular biology of aflatoxin biosynthesis. Chemical Reviews 97(7), 2537-2556.

Miraglia, M., Marvin, H.J.P., Kleter, G.A., Battilani, P., Brera, C., Coni, E., Cubadda, F., Croci, L., De Santis, B., Dekkers, S., Filippi, L., Hutjes, R.W.A., Noordam, M.Y., Pisante, M., Piva, G., Prandini, A., Toti, L., van den Born, G.J., Vespermann, A., 2009. Climate change and food safety: an emerging issue with special focus on Europe. Food Chemistry and Toxicology 47, 1009-1021.

Monson, M.S., Coulombe, R.A., Reed, K.M., 2015. Aflatoxicosis: Lessons from toxicity and responses to aflatoxin B1 in poultry. Agriculture 5(3), 742-777.

Moon, Y.-S., Kim, H.-M., Chun, H.S., Lee, S.-E., 2018. Organic acids suppress aflatoxin production via lowering expression of aflatoxin biosynthesis-related genes in Aspergillus flavus. Food Control 88, 207-216.

Moreno, E.C., Garcia, G.T., Ono, M.A., Vizoni, É., Kawamura, O., Hirooka, E.Y., Ono, E.Y.S., 2009. Co-occurrence of mycotoxins in corn samples from the Northern region of Paraná State, Brazil. Food Chemistry 116(1), 220-226.

Mousa, W., Ghazali, F.M., Jinap, S., Ghazali, H.M., Radu, S. 2013. Modeling growth rate and assessing aflatoxins production by A. flavus as a function of water activity and temperature on polished and brown rice. Journal of Food Science 78(1), 56-63.

Murjani, G., 2003. Chronic aflatoxicosis in fish and its relevance to human health. Central Institute of Freshwater Aquaculture, India.

Mutegi, C.K., Ngugi, H.K., Hendriks, S.L., Jones, R.B., 2009. Prevalence and factors associated with aflatoxin contamination of peanuts from Western Kenya, International Journal of Food Microbiology 130, 27-34.

Mutegi, C.K., Ngugi, H.K., Hendriks, S.L., Jones, R.B., 2012. Factors associated with the incidence of $A$. section Flavi and aflatoxin contamination of peanuts in the Busia and Homa bay districts of western Kenya. Plant Pathology 61, 1143-1153.

Mwakinyali, S.E., Ding, X., Ming, Z., Tong, W., Zhang, Q., Li, P., 2019. Recent development of aflatoxin contamination biocontrol in agricultural products. Biological Control 128, 31-39.

Narasaiah, K.V., Sashidhar, R.B., Subramanyam, C., 2006. Biochemical analysis of oxidative stress in the production of aflatoxin and its precursor intermediates. Mycopathologia 162, 179-189.

Newberne, P.M., 1965. Carcinogenicity of AflatoxinContaminated Pea nut meal. In: G. N. Wogan (ed.), Mycotoxins in Foodstuffs,. Cambridge, Mass.: MIT Press, pp. 187-208

Newberne, P.M., Butler, W.H., 1969. Acute and chronic effects of aflatoxin on the liver of domestic and laboratory animals: a review. Cancer Research 29(1), 236-250.

Ngindu, A., Kenya, P., Ocheng, D., Omondi, T., Ngare, W., Gatei, D., Johnson, B., Ngira, J., Nandwa, H., Jansen, A., Kaviti, J.,
1982. Outbreak of acute hepatitis caused by aflatoxin poisoning in Kenya. The Lancet 319(8285), 1346-1348.

Njumbe Ediage, E., Hell, K., De Saeger, S., 2014. A comprehensive study to explore differences in mycotoxin patterns from agro-ecological regions through maize, peanut, and cassava products: a case study, Cameroon. Journal of Agricultural and Food Chemistry 62(20), 4789-4797.

OBrian, G.R., Georgianna, D.R., Wilkinson, J.R., Yu, J., Abbas, H.K., Bhatnagar, D., Cleveland, T.E., Nierman, W.C., Payne, G.A., 2007. The effect of elevated temperature on gene transcription and aflatoxin biosynthesis. Mycologia 99, 232-239.

Okoth, S.A., Ohingo, M., 2004. Dietary aflatoxin exposure and impaired growth in young children from Kisumu District, Kenya: cross sectional study. African Journal of Health Science 11, 43-54

Olinda, R.G., Lima, J.M., de Lucena, R.B., do Vale, A.M., Batista, J.S., de Barros, C.S.L., Riet-Correa, F. and Dantas, A.F.M., 2016. Acute aflatoxicosis in swines in Northeastern Brazil. Acta Scientiae Veterinariae 44(Supplement).

Orsi, R.B., Oliveira, C.A.F., Dilkin, P., Xavier, J.G., Direito, G.M., Correa, B., 2007. Effects of oral administration of aflatoxin B1 and fumonisin B1 in rabbits (Oryctolagus cuniculus). Chemico-Biological Interactions 170, 201208.

Ortiz, M.P., Barros, G.G., Reynoso, M.M., Torres, A.M., Chulze, S. N., Ramirez, M.L., 2011. Soil populations of $A$. section Flavi from the main and new peanut growing areas in Argentina. ISM Conference 2011 "Strategies to reduce the impact of mycotoxins in Latin America in a global context". Abstract Book.

Oruc, H.H., Cengiz, M., Kalkanli, O., 2006. Comparison of aflatoxin and fumonisin levels in maize grown in Turkey and imported from the USA. Animal Feed Science and Technology 128, 337-341.

Osman, N., El-sabban, F.F., Khawli, A.A., Mensah-Brown, E.P.K., 2004. Effect of foodstuff contamination by aflatoxin on the one-humped camel (Camelus dromedarius) in Al Ain, United Arab Emirates. Australian Veterinary Journal 82(12), 759-761.

Ozias-Akins, P., Yang, H., Gill, R., Fan, H., Lynch, R.E., 2002. Reduction of aflatoxin contamination in peanut: A genetic engineering approach. Crop Biotechnology 829, 151-160.

Pandey, I., Chauhan, S.S., 2007. Studies on production performance and toxin residues in tissues and eggs of layer chickens fed on diets with various concentrations of aflatoxin AFB1. British Poultry Science 48(6), 713-723.

Paterson, R., Lima, N., 2010. How will climate change affect mycotoxins in food? Food Research International 43, 1902-1914.

Paterson, R., Lima, N., 2011. Further mycotoxin effects from climate change. Food Research International 44, 25552566. 
Patterson, D.S.P., 1973. Metabolism as a factor in determining the toxic action of the aflatoxins in different animal species. Food and Cosmetics Toxicology 11(2), 287-294.

Patterson, D.S.P., Roberts, B.A., 1970. The formation of aflatoxins B2a and G2a and their degradation products during the in vitro detoxification of aflatoxin by livers of certain avian and mammalian species. Food and Cosmetics Toxicology 8(5), 527-538.

Payne, G.A., Brown, M.P., 1998. Genetics and physiology of aflatoxin biosynthesis. Annual Review of Phytopathology 36, 329-362.

Payne, G.A., Hagler, W.M., 1983. Effect of specific amino acids on growth and aflatoxin production by $A$. parasiticus and A. flavus in defined media. Applied and Environmental Microbiology 46(4), 805-812.

Peers, F.G., Linsell, C.A., 1977. Dietary aflatoxins and human primary liver cancer. Annales de la Nutrition et de L'alimentation 31, 1005-17

Peng, Z., Chen, L., Zhu, Y., Huang, Y., Hu, X., Wu, Q., Nüssler, A.K., Liu, L., Yang, W., 2018. Current major degradation methods for aflatoxins: A review. Trends in Food Science \& Technology 80, 155-166.

Pereyra, M.L.G., Carvalho, E.C., Tissera, J.L., Keller, K.M., Magnoli, C.E., Rosa, C.A., Dalcero, A.M., Cavaglieri, L.R., 2008. An outbreak of acute aflatoxicosis on a chinchilla (Chinchilla lanigera) farm in Argentina. Journal of Veterinary Diagnostic Investigation, 20(6): 853-856.

Piekkola, S., Turner, P.C., Abdel-Hamid, M., Ezzat, S., El-Daly, M., El-Kafrawy, S., Savchenko, E., Poussa, T., Woo, J.C.S., Mykkänen, H. and El-Nezami, H., 2012. Characterisation of aflatoxin and deoxynivalenol exposure among pregnant Egyptian women. Food Additives \& Contaminants: Part A 29(6), 962-971.

Pier, A.C., 1992. Major biological consequences of aflatoxicosis in animal production. Journal of Animal Science 70(12), 3964-3967.

Price, M.S., Yu, J., Nierman, W.C., Kim, H.S., Pritchard, B., Jacobus, C.A., Bhatnagar, D., Cleveland, T.E., Payne, G.A., 2006. The aflatoxin pathway regulator AflR induces gene transcription inside and outside of the aflatoxin biosynthetic cluster. FEMS Microbiology Letters 255(2), 275-279.

Priyadarshini, E., Tulpule, P.G., 1980. Effect of free fatty acids on aflatoxin production in a synthetic medium. Food and Cosmetics Toxicology 18(4), 367-369.

Quist, C.F., Bounous, D.I., Kilburn, J.V., Nettles, V.F., Wyatt, R.D., 2000. The effect of dietary aflatoxin on wild turkey poults. Journal of Wildlife Diseases 36(3), 436-444.

Quist, C.F., Howerth, E.W., Fischer, J.R., Wyatt, R.D., Miller, D.M., Nettles, V.F., 1997. Evaluation of low-level aflatoxin in the diet of white-tailed deer. Journal of Wildlife Diseases 33(1), 112-121.

Raju, M.V.L.N., Devegowda, G., 2000. Influence of esterifiedglucomannan on performance and organ morphology, serum biochemistry and haematology in broilers exposed to individual and combined mycotoxicosis (aflatoxin, ochratoxin and T-2 toxin). British Poultry Science 41(5), 640-650.

Ramsdell, H.S., Eaton, D.L., 1990. Species susceptibility to Aflatoxin B1 carcinogenesis. Cancer Research 50, 615620.

Ratnavathi, C.V., Komala, V.V., Kumar, B.S.V., Das, I.K., Patil, J.V., 2012. Natural occurrence of aflatoxin B1 in sorghum grown in different geographical regions of India. Journal of the Science of Food and Agriculture 92(12), 24162420 .

Rawal, S., Kim, J.E., Coulombe, R., 2010. Aflatoxin B1 in poultry: Toxicology, metabolism and prevention. Research in Veterinary Science 89, 325-331.

Reddy, T.V., Viswanathan, L., Venkitasubramanian, T.A., 1979. Factors affecting aflatoxin production by $A$. parasiticus in a chemically defined medium. Journal of General Microbiology 114, 409-413.

Reddy, T.Y., Sulochanamma, B.N., Subramanyam, A., Balaguravaiah, D., 2003. Influence of weather, dry spells and management practices on aflatoxin contamination in groundnut. Indian Phytopathology 56, 262-265.

Reib, J., 1982. Development of $A$. parasiticus and formation of aflatoxin $B_{1}$ under the influence of conidiogenesis affecting compounds. Archives of Microbiology 133, 236238.

Reiss, J., 1972a. Comparative investigations on the toxicity of some mycotoxins to the larvae of the brine shrimp (Artemia salina L.). Zentralblatt fur Bakteriologie, Parasitenkunde, Infektionskrankheiten und Hygiene. Erste Abteilung Originale. Reihe B: Hygiene, Praventive Medizin 155(5), 531-534.

Reiss, J., 1972b. Toxic effects of the mycotoxins aflatoxin B 1, rubratoxin B, patulin, and diacetoxyscirpenol on the crustacean Cyclops fuscus. Journal-Association of Official Analytical Chemists 55(4), 895.

Reverberi, M., Zjalic, S., Ricelli, A., Fabbri, A. A., Fanelli, C., 2006. Oxidant/antioxidant balance in $A$. parasiticus affects aflatoxin biosynthesis. Mycotoxin Research 22(1), 39-47.

Ribeiro, D.H., Ferreira, F.L., Da Silva, V.N., Aquino, S., Corrêa, B., 2010. Effects of aflatoxin B1 and fumonisin B1 on the viability and induction of apoptosis in rat primary hepatocytes. International Journal of Molecular Sciences 11(4), 1944-1955.

Richard, E., Heutte, N., Bouchart, V., Garon, D., 2009. Evaluation of fungal contamination and mycotoxin production in maize silage. Animal Feed Science and Technology 148(2), 309-320.

Robens, J., Cardwell, K., 2003. The costs of mycotoxin management to the USA: Management of aflatoxins in the United States. Journal of Toxicology, Toxin Reviews 2-3, 143-156.

Robens, J.F., Richard, J.L., 1992. Aflatoxins in animal and human health. Review in Environmental and Contamination and Toxicology 127, 69-94. 
Robinson, R.M., Ray, A.C., Reagor, J.C., Holland, L.A., 1982. Waterfowl mortality caused by aflatoxicosis in Texas. Journal of Wildlife Diseases 18(3), 311-313.

Rodrigues, I., Naehrer, K., 2012. A three-year survey on the worldwide occurrence of mycotoxins in feedstuffs and feed. Toxins 4(9), 663-675.

Royes, J.B., Yanong, R.P., 2002. Molds in fish feeds and aflatoxicosis. Copyright by the University of Florida, Institute of Agricultural Science (UF/IFAS).

Roze, L.V., Chanda, A., Wee, J., Awad, D., Linz, J.E., 2011. Stress-related transcription factor AtfB integrates secondary metabolism with oxidative stress response in aspergilli. Journal of Biological Chemistry 286(40), 35137-35148.

Sadeghi N, Oveisi M, Jannat B, Hajimahmoodi M, Bonyani H, Jannat F., 2009. Incidence of aflatoxin M 1 in human breast milk in Tehran, Iran. Food Control 20, 75-78

Sahoo, P.K., Mukherjee, S.C., Jain, A.K., Mukherjee, A., 2003. Histopathological and Electron Microscopic Studies of Gills and Opisthonephros of Rohu, Labeo rohita to Acute and Subchronic Aflatoxin B 1 Toxicity. Asian Fisheries Science 16(3/4), 257-268.

Salem, R., El-Habashi, N., Fadl, S.E., Sakr, O.A., Elbialy, Z.I., 2018. Effect of probiotic supplement on aflatoxicosis and gene expression in the liver of broiler chicken. Environmental Toxicology and Pharmacology 60, 118127.

Salmon, W.D., Newberne, P.M., 1963. Occurrence of hepatomas in rats fed diets containing peanut meal as a major source of protein. Cancer Research 23(4 Part 1), 571-575.

Sanders, T.H., Cole, R.J., Blankenship, P.D., Hill, R.A., 1985. Relation of environmental stress duration to $A$. flavus invasion and aflatoxin production in preharvest peanuts. Peanut Science 12(2), 90-93.

Sangare-Tigori, B., Moukha, S., Kouadio, H.J., Betbeder, A.M., Dano, D.S., Creppy, E.E., 2006. Co-occurrence of aflatoxin B1, fumonisin B1, ochratoxin A and zearalenone in cereals and peanuts from Côte d'Ivoire. Food Additives and Contaminants, 23(10), 1000-1007.

Scheidegger, K.A., Payne, G.A., 2003. Unlocking the secrets behind secondary metabolism: a review of $A$. flavus from pathogenicity to functional genomics. Journal of Toxicology: Toxin Reviews 22(2-3), 423-459.

Schmidt-Heydt, M., Rufer, C.E., Abdel-Hadi, A., Magan, N., Geisen, R., 2010. The production of aflatoxin B1 or G1 by A. parasiticus at various combinations of temperature and water activity is related to the ratio of aflS to aflR expression. Mycotoxin Research 26, 241-246.

Schoental, R., 1967. Aflatoxins. Annual Review of Pharmacology 7(1), 343-356.

Schothorst, R.C., van Egmond, H.P., 2004. Report from SCOOP task 3.2. 10 "collection of occurrence data of Fusarium toxins in food and assessment of dietary intake by the population of EU member states": subtask: trichothecenes. Toxicology Letters 153(1), 133-143.
Sedmikova, M., Reisnerova, H., Dufkova, Z., Barta, I., Jilek, F., 2001. Potential hazard of simultaneous occurrence of aflatoxin B-1 and ochratoxin A. Veterinarni MedicinaCzech 46, 169-174.

Sepahdari, A., Ebrahimzadeh Mosavi, H.A., Sharifpour, I., Khosravi, A., Motallebi, A.A., Mohseni, M.A.H.M.O.U.D., Kakoolaki, S., Pourali, H.R., Hallajian, A., 2010. Effects of different dietary levels of AFB1 on survival rate and growth factors of Beluga (Huso huso). Iranian Journal of Fisheries Sciences 9(1), 141-150.

Shahbazi, Y., Shavisi, N., 2019. Occurrence of aflatoxins and ochratoxin A in Gaz, a traditional Persian confection, and its ingredients. Food Control 98, 107-112.

Shane, S.H., 1994. Economic issues associated with aflatoxins. In: Eaton, D.L., Groopman, J.D. (Eds.), The Toxicology of Aflatoxins: Human Health, Veterinary, and Agricultural Significance. Academic Press, San Diego, pp. 513-527.

Shank, R.C., Bourgeois, C.H., Keschamras, N., Chandavimol, P., 1971. Aflatoxins in autopsy specimens from Thai children with an acute disease of unknown aetiology. Food Cosmetics and Toxicology 9, 501-507.

Shen, H.M., Ong, C.N., Shi, C.Y., 1995. Involvement of reactive oxygen species in aflatoxin B1-induced cell injury in cultured rat hepatocytes. Toxicology 99, 115-123.

Shetty, P.H., Bhat, R.V., 1997. Natural occurrence of fumonisin B1 and its co-occurrence with aflatoxin B1 in Indian sorghum, maize, and poultry feeds. Journal of Agricultural and Food Chemistry 45, 2170-2173.

Shirima, C.P., Kimanya, M.E., Kinabo, J.L., Routledge, M.N., Srey, C., Wild, C.P., Gong, Y.Y., 2013. Dietary exposure to aflatoxin and fumonisin among Tanzanian children as determined using biomarkers of exposure. Molecular Nutrition \& Food Research 57(10), 1874-1881.

Shuaib, F.M., Jolly, P.E., Ehiri, J.E., Yatich, N., Jiang, Y., Funkhouser. E., Person, S.D., Wilson, C., Ellis, W.O., Wang, J.S., Williams, J.H., 2010. Association between birth outcomes and aflatoxin B1 biomarker blood levels in pregnant women in Kumasi, Ghana. Tropical Medicine and International Health 15,160-167.

Singh, P., Cotty, P.J., 2019. Characterization of Aspergilli from dried red chilies (Capsicum spp.): Insights into the etiology of aflatoxin contamination. International Journal of Food Microbiology 289, 145-153.

Smerak, P., Barta, I., Polivkova, Z., Bartova, J., Sedmikova, M., 2001. Mutagenic effects of selected tichothecene mycotoxins and their combinantions with aflatoxin B1. Czech Journal of Food Science 19, 90-96.

Spanjer, M.C., 2019. Occurrence \& Risk of Aflatoxins in Food and Feed, in: Melton, L., Shahidi, F., Varelis, P. (Eds.), Encyclopedia of Food Chemistry. Content Repository Only!, Oxford, pp. 424-427.

Streit, E., Naehrer, K., Rodrigues, I., Schatzmayr, G., 2013. Mycotoxin occurrence in feed and feed raw materials worldwide: long-term analysis with special focus on Europe and Asia. Journal of the Science of Food and Agriculture 93(12), 2892-2899. 
Streit, E., Schatzmayr, G., Tassis, P., Tzika, E., Marin, D., Taranu, I., Tabuc, C., Nicolau, A., Aprodu, I., Puel, O., Oswald, I.P., 2012. Current situation of mycotoxin contamination and co-occurrence in animal feed-Focus on Europe. Toxins 4(10), 788-809.

Strosnider, H., Azziz-Baumgartner, E., Banziger, M., Bhat, R.V., Breiman, R., Brune, M.N., DeCock, K., Dilley, A., Groopman, J., Hell, K., Henry, S.H., Jeffers, D., Jolly, C., Jolly, P., Kibata, G.N., Lewis, L., Liu, X., Luber, G., McCoy, L., Mensah, P., Miraglia, M., Misore, A., Njapau, H., Ong, C.N., Onsongo, M.T., Page, S.W., Park, D., Patel, M., Phillips, T., Pineiro, M., Pronczuk, J., Rogers, H.S., Rubin, C., Sabino, M., Schaafsma, A., Shephard, G., Stroka, J., Wild, C., Williams, J.T., Wilson, D., 2006. Workgroup report: public health strategies for reducing aflatoxin exposure in developing countries. Environmental Health Perspectives 114, 1898-1903.

Suliman, H.B., Mohamed, A.F., Awadelsied, N.A., Shommein, A.M., 1987. Acute mycotoxicosis in sheep: Field cases. Veterinary and Human Toxicology 29, 241-243.

Sun, G., Wang, S., Hu, X., Su, J., Zhang, Y., Xie, Y., Zhang, H., Tang, L., Wang, J.S., 2011. Co-contamination of aflatoxin B1 and fumonisin B1 in food and human dietary exposure in three areas of China. Food Additives and Contaminants 28(4), 461-470.

Thakare, D., Zhang, J., Wing, R.A., Cotty, P. J., Schmidt, M.A., 2017. Aflatoxin-free transgenic maize using host-induced gene silencing. Science Advances 3(3), e1602382.

Theumer, M.G., Canepa, M.C., Lopez, A.G., Mary, V.S., Dambolena, J.S., Rubinstein, H.R., 2010. Subchronic mycotoxicoses in Wistar rats: assessment of the in vivo and in vitro genotoxicity induced by fumonisins and aflatoxin B1, and oxidative stress biomarkers status. Toxicology 268, 104-110.

Theumer, M.G., Lopez, A.G., Aoki, M.P., Canepa, M.C., Rubinstein, H.R., 2008. Subchronic mycotoxicoses in rats. Histopathological changes and modulation of the sphinganine to sphingosine (Sa/So) ratio imbalance induced by Fusarium verticillioides culture material, due to the coexistence of aflatoxin B1 in the diet. Food and Chemical Toxicology 46, 967-977.

Theumer, M.G., Lopez, A.G., Masih, D.T., Chulze, S.N., Rubinstein, H.R., 2003. Immunobiological effects of AFB1 and AFB1eFB1 mixture in experimental subchronic mycotoxicoses in rats. Toxicology 186, 159-170.

Torres, A.M., Barros, G.G., Palacios, S.A., Chulze, S.N., Battilani, P., 2014. Review on pre- and post-harvest management of peanuts to minimize aflatoxin contamination. Food Research International 62, 11-19.

Trung, T., Tabuc, C., Bailly, S., Querin, A., Guerre, P., Bailly, J., 2008. Fungal mycoflora and contamination of maize from Vietnam with aflatoxin B 1 and fumonisin B 1. World Mycotoxin Journal 1, 87-94.

Tsounidi, D., Koukouvinos, G., Petrou, P., Misiakos, K., Zisis, G., Goustouridis, D., Raptis, I., Kakabakos, S.E., 2019. Rapid and sensitive label-free determination of aflatoxin M1 levels in milk through a White Light Reflectance
Spectroscopy immunosensor. Sensors and Actuators B: Chemical 282, 104-111.

Tuan, N.A., Grizzle, J.M., Lovell, R.T., Manning, B.B., Rottinghaus, G.E., 2002. Growth and hepatic lesions of Nile tilapia (Oreochromis niloticus) fed diets containing aflatoxin B1. Aquaculture 212, 311-319.

Turner, P.C., Collinson, A.C., Cheung, Y.B., Gong, Y., Hall, A.J., Prentice, A.M., Wild, C.P., 2007. Aflatoxin exposure in utero causes growth faltering in Gambian infants. International Journal of Epidemiology 36(5), 1119-1125.

Udomkun, P., Wiredu, A. N., Nagle, M., Bandyopadhyay, R., Müller, J., Vanlauwe, B., 2017. Mycotoxins in Sub-Saharan Africa: Present situation, socio-economic impact, awareness, and outlook. Food Control 72, 110-122.

Ueng, Y.F., Shimada, T., Yamazaki, H., Guengerich, F.P., 1995 Oxidation of aflatoxin B1 by bacterial recombinant human cytochrome P450 enzymes. Chemical Research in Toxicology 8, 218-225.

Umar, S., Munir, M.T., Shah, M.A., Shahzad, M., Khan, R.A., Sohoo, M.U.R., Khan, A.U., Ameen, K., Rafia-Munir, A., Saleem, F., 2015. Outbreak of aflatoxicosis on a local cattle farm in Pakistan. Veterinaria 3, 13-17

Uriah, N., Ibeh, I. N., Oluwafemi, F., 2001. A Study on the Impact of Aflatoxin on Human Reproduction. Laboratory Report. African Journal of Reproductive Health/La Revue Africaine de la Santé Reproductive 5(1), 106-110.

Van Rensburg, S.J., Cook-Mozaffari, P., Van Schalkwyk, D.J., Van der Watt, J.J., Vincent, T.J., Purchase, IF., 1985. Hepatocellular carcinoma and dietary aflatoxin in Mozambique and Transkei. British Journal of Cancer 51, 713-726

Vardon, P., McLaughlin, C., Nardinelli, C., 2003. Potential economic costs of mycotoxins in the United States. In: Council for Agricultural Science and Technology (CAST). Mycotoxins: risks in plant, animal, and human systems; Task Force Report No. 139: Ames, Iowa.

Veldman, A., Meijs, J.A.C., Borggreve, G.J., Heeres-Van der Tol, J.J., 1992. Carry-over of aflatoxin from cows' food to milk. Animal Production 55(02), 163-168.

Verma, D.S., 2005. Mycotoxin biotransformation: latest approach to well-known problem on Indian feed industry. Poultry World 1(9), 34-35.

Vidal, A., Marín, S., Sanchis, V., De Saeger, S., De Boevre, M., 2018. Hydrolysers of modified mycotoxins in maize: $\alpha-$ Amylase and cellulase induce an underestimation of the total aflatoxin content. Food Chemistry 248, 86-92.

Vidhyasekaran, P., Lalithakumari, D., Govindaswamy, C.V., 1972. Production of a phytoalexin in groundnut due to storage fungi. Indian Phytopathology 25, 240-245.

Vilar, M.S., Kuilman-Wahls, M.E., Fink-Gremmels, J., 2003. Inhibition of aflatoxin $\mathrm{B}_{1}$ mutagenicity by cyclopiazonic acid in the presence of human liver preparations. Toxicology Letters 143(3), 291-299.

Waliyar, F., Kumar, P.L., Ntare, B.R., Diarra, B., Kodio, O., 2008. Pre-and post-harvest management of aflatoxin contamination in peanuts. In: Leslie, J.F., Bandyopadhyay, R. and Visconti, A. (eds.) Mycotoxins: detection methods, 
management, public health and agricultural trade. CABI Publishing, Wallingford, UK, pp. 209-218.

Wang, G., Xi, Y., Lian, C., Sun, Z., Zheng, S., 2019a. Simultaneous detoxification of polar aflatoxin B1 and weak polar zearalenone from simulated gastrointestinal tract by zwitterionic montmorillonites. Journal of Hazardous Materials 364, 227-237.

Wang, J.S., Abubaker, S., He, X., Sun, G., Strickland, P.T., Groopman, J.D., 2001. Development of aflatoxin B1-lysine adduct monoclonal antibody for human exposure studies. Applied Environmental Microbiology 67 (6), 2712-2717.

Wang, J.S., Groopman, J.D., 1999. DNA damage by mycotoxins. Mutation Research 424, 167-181.

Wang, L., Zhu, F., Chen, M., Zhu, Y., Xiao, J., Yang, H., Chen, X., 2019b. Rapid and visual detection of aflatoxin B1 in foodstuffs using aptamer/G-quadruplex DNAzyme probe with low background noise. Food Chemistry 271, 581587.

Warth, B., Parich, A., Atehnkeng, J., Bandyopadhyay, R., Schuhmacher, R., Sulyok, M., and Krska, R. (2012). Quantitation of mycotoxins in food and feed from Burkina Faso and Mozambique using a modern LCMS/MS multitoxin method. Journal of Agricultural and Food Chemistry 60(36), 9352-9363.

Wild, C.P., Hudson, G.J., Sabbioni, G., Chapot, B., Hall, A.J., Wogan, G.N., Whittle, H., Montesano, R., Groopman, J.D., 1992. Dietary intake of aflatoxins and the level of albumin-bound aflatoxin in peripheral blood in The Gambia, West Africa. Cancer Epidemiology Biomarkers and Prevention 1, 229-234.

Wild, C.P., Pisani, P., 1998. Carcinogen DNA and protein adducts as biomarkers of human exposure in environmental cancer epidemiology. Cancer Detection and Prevention 22, 273- 83.

Wilkinson, J.R., Yu, J., Bland, J.M., Nierman, W.C., Bhatnagar, D., Cleveland, T.E., 2007. Amino acid supplementation reveals differential regulation of aflatoxin biosynthesis in A. flavus NRRL 3357 and A. parasiticus SRRC 143. Applied Microbiology Biotechnology 74, 1308-1319.

Williams, J. H., Phillips, T.D., Jolly, P.E., Stiles, J.K., Jolly, C.M., Aggarwal, D. 2004. Human aflatoxicosis in developing countries: a review of toxicology, exposure, potential health consequences, and interventions. The American Journal of Clinical Nutrition 80(5), 1106-1122.

Wiseman, M.O., Price, R. L., Lightner, D. V., Williams, R.R., 1982. Toxicity of aflatoxin B1 to penaeid shrimp. Applied and Environmental Microbiology 44(6), 1479-1481.

Wogan, G.N., 1969. Naturally Occurring Carcinogens in Foods1. In Carcinogenesis and Carcinogen Testing. Karger Publishers, 11, 134-162.

Woloshuk, C.P., Cavaletto, J.R., Cleveland, T.E., 1997. Inducers of aflatoxin biosynthesis from colonized maize kernels are generated by an amylase activity from A. flavus. Phytopathology 87, 164-169.

Wong, Z.A., Hsieh, D. P., 1980. The comparative metabolism and toxicokinetics of aflatoxin $\mathrm{B}_{1}$ in the monkey, rat, and mouse. Toxicology and Applied Pharmacology 55(1), 115-125.

Wotton, H.R., Strange, R.N., 1985. Circumstantial evidence for phytoalexin involvement in the resistance of peanuts to A. flavus. Journal of General Microbiology 131, 487-494.

Wright, M.S., Greene-McDowelle, D.M., Zeringue, H.J., Bhatnagar, D., Cleveland, T.E., 2000. Effects of volatile aldehydes from A.-resistant varieties of corn on $A$. parasiticus growth and aflatoxin biosynthesis. Toxicon 38(9), 1215-1223.

$\mathrm{Wu}, \mathrm{F} ., 2006$. Mycotoxin reduction in Bt corn: Potential economic, health and regulatory impacts. Transgenic Research 15, 277-289.

Wu, F., Guclu, H., 2012. Aflatoxin regulations in a network of global maize trade. PloS One 7(9), e45151.

Wu, T.-S., Cheng, Y.-C., Chen, P.-J., Huang, Y.-T., Yu, F.-Y., Liu, B.-H., 2019. Exposure to aflatoxin B1 interferes with locomotion and neural development in zebrafish embryos and larvae. Chemosphere 217, 905-913.

Wyllie, T.D., Morehouse, L.G., 1978. Mycotoxic fungi, mycotoxins, mycotoxicoses. In: Wyllie TD, Morehouse LG (eds) An encyclopedic handbook; Marcel Dekker, New York.

Xie, H., Dong, J., Duan, J., Hou, J., Ai, S., Li, X., 2019a. Magnetic nanoparticles-based immunoassay for aflatoxin B1 using porous g-C3N4 nanosheets as fluorescence probes. Sensors and Actuators B: Chemical 278, 147-152.

Xie, Y., Wang, W., Zhang, S., 2019b. Purification and identification of an aflatoxin B1 degradation enzyme from Pantoea sp. T6. Toxicon 157, 35-42.

Xu, J.-J., Chen, Q., Cai, Z.-X., Ren, Y.-P., Zhao, Y.-F., Cheng, J., Huang, B.-F., 2018. A feasibility study of producing a peanut oil matrix candidate reference material and its application to support monitoring of aflatoxins statues for public health purposes. Food Chemistry 268, 395401.

Yabe, K., Nakajima, H., 2004. Enzyme reactions and genes in aflatoxin biosynthesis. Applied Microbiology and Biotechnology 64, 745-755.

Yalagod Shivasharanappa, G., Mundas, S., Rao, D.G.K., Tikare, V., Shridhar, N.B., 2013. Histopathological changes in pigs exposed to aflatoxin B1 during pregnancy. Indian Journal of Animal Research, 47(5), 386-391.

Yates, I.E., Cole, R.J., Giles, J.L., Dorner, J.W., 1987. Interaction of aflatoxin $\mathrm{B}_{1}$ and cyclopiazonic acid toxicities. Molecular Toxicology 1, 95-106.

$\mathrm{Yu}, \mathrm{J} ., 2012$. Current understanding on aflatoxin biosynthesis and future perspective in reducing aflatoxin contamination. Toxins 4, 1024-1057.

Yu, J., Chang, P.K., Ehrlich, K.C., Cary, J.W., Bhatnagar, D., Cleveland, T.E., Payne, G.A., Linz, J.E., Woloshuk, C.P., Bennett, J.W., 2004. Clustered pathway genes in aflatoxin biosynthesis. Applied and Environmental Microbiology, 70(3), 1253-1262.

Yu, J., Mohawed, S. M., Bhatnagar, D., Cleveland, T. E., 2003. Substrate-induced lipase gene expression and aflatoxin 
production in A. parasiticus and A. flavus. Journal of Applied Microbiology 95(6), 1334-1342.

Yu, X., Li, Z., Zhao, M., Lau, S.C.S., Ru Tan, H., Teh, W.J., Yang, H., Zheng, C., Zhang, Y., 2019. Quantification of aflatoxin B1 in vegetable oils using low temperature clean-up followed by immuno-magnetic solid phase extraction. Food Chemistry 275, 390-396.

Zain, M. E., 2011. Impact of mycotoxins on humans and animals. Journal of Saudi Chemical Society 15, 129e144.

Zeng, Z.-Z., Jiang, W.-D., Wu, P., Liu, Y., Zeng, Y.-Y., Jiang, J., Kuang, S.-Y., Tang, L., Zhou, X.-Q., Feng, L., 2019. Dietary aflatoxin $\mathrm{B} 1$ decreases growth performance and damages the structural integrity of immune organs in juvenile grass carp (Ctenopharyngodon idella). Aquaculture 500, 1-17.

Zhao, F., Tian, Y., Shen, Q., Liu, R., Shi, R., Wang, H., Yang, Z., 2019. A novel nanobody and mimotope based immunoassay for rapid analysis of aflatoxin B1. Talanta 195, 55-61.

Zhou, J., Tang, L., Wang, J.-S., 2019. Assessment of the adverse impacts of aflatoxin B1 on gut-microbiota dependent metabolism in F344 rats. Chemosphere 217, 618-628.

Visit us at: http://bosaljournals.com/chemint/

Submissions are accepted at: editorci@bosaljournals.com 